\title{
Joachim Perels Die Restauration der Rechtslehre nach 1945
}

Nach der militärischen Niederwerfung des nationalsozialistischen Terrorstaates wurde der weitüberwiegende Teil seiner Trägerschichten in der Justiz und in den juristischen Fakultäten in die neu konstimierte demokratische Verfassungsordnung Westdeutschlands übernommen. ${ }^{1}$ Daß dies die Entwicklung demokracischen Rechtsdenkens mit einer strukturellen Hypothek belastece, rückte kaum ins allgemeine Bewußtsein. Zwar existieren einige nützliche Detailuntersuchungen zum Problem der personellen Kontinuität der juriscischen Funktionselite des Dritten Reiches und zur Frage der Kontinuität einzelner Rechrskonscruktionen im öffertlichen Recht und im Arbcitsrecht.' Es fehlt aber eine Gesamtanalyse, welche die aus diesen Kontinuitärsstrukcuren folgenden Konsequenzen für die Ausbildung eines umfassenden bürgerlich-restaurativen Rechrsbegriffs und einer ihm entsprechender Rechtspraxis systemacisch behandelte.' Daß eine derartige Untersuchung bisher nicht existiert, hängt mit erfolgreichen, noch immer fortwirkenden Verdrängungsprozessen zusammen. Die crnstliche Erörterung des Problems, daß die juriscischen Interpretacionseliten der Diktatur zu denen der Demokratie wurden, hätte zu einem Legitimationsverlust der rechtlichen Entscheidungsprozesse der frühcn Bundesrepublik geführc. So blieb diese Fragestellung ein Tabu und damit auch der Forschung nahezu durchgängig entzogen.

Wenn von der restaurativen Rechtslehre gesprochen wird, so nötigt die jüngere Literatur, die den Restauracionsbegriff für die Kennzeichnung des politischen und gesellschaftlichen Systcms in Westdeutschland mit der Begründung verwirft, mit ihm werde der demokratische und rechisstaacliche Neubeginn der Bundesrepublik

I J.R. Wenzlau, Der Wiederaufbau der Justiz, in Nordwestdeutschland 1949-1949, Königstein 1979; J. Feest, Die Bundesrichter. Herkunft, Karriere und Auswahl der juristischen Elice, 1n: W. Zapl (Hg.), Beiuäge zur Analyse der deutschen Oberschicht, 2, Aufl., München 1965, S. 95 ff.; R. Wahsner, Das Arbeissrechtskanell. Die Restauracion des kapitalisuischen Arbeitsreches in Westdeuschland nach r 945. KJ H. 4/1974, S. 369 ff.; H. Fangmann, Die Restauracion der hereschenden Staztsrechiswissenschaft nach 1945, in: U. Rcifncr (Hg.), Das Recht des Unrechessiaates, Frankfurn a. M. 1981, S. 2 r If.

$2 V_{g l}$. ezwa D. Deiseroih, Kontinuicätsprobleme der deutschen S(azesreclieslehre(r)?, in: Abendrouh, Blanke, Preuß u. n., Ordnungsmache? Über das Verhälenis von Legalität, Konsens und Herrschafi, hrse. von D. Deiseroth, F. Hase und K.-H. Ladeur, Franklun a. M. 1981, S. 8, If.; J. Scilcri, Vercinigungsfreiheit und hoheitiche Verrulsceklärungen, in: J. Perels ( $\left.\mathrm{Hg}_{\mathrm{g}}\right)$, Grundrechte als Fundament der Demokratic, Frankfurn 2. M. 1979, S. 178 Anm. 29; W. Abendroth. Innergewerkschaftliche Willensbildung, Urabscimmung und "Kamplmaßnahme* (1959), in: ders., Antagonistische Gesellschaft und politische Demoluatic, z. Aufl. Ncuwied 1972, S. 25 Iff. (257-260); Th. Ramm, Sozialadaquanztheorie und freiheilich-30zialer Rechusstaat, AuR 1966, S. 1636 .

$3 \mathrm{Vgl}$ aber de wichtigen Vorarbeiten: M. Kirn, Verfassungsumsturz oder Rechtskontinuität? Dic Stellung der Jurisprudenz nach I94s zum Driten Reich, insbesondere die Konflikte um die Kantinuititi der Bcamtenrechte und Arr. 131 Grandgesetx, Bcrlin 1972: B. Distelkamp, Rechts- und veriassungsgeschicheliche Probleme zur Frühgcschichte der Bundesrepublik Deutschland, JuS 1980, S. 40 I If., $4^{8}$ I If., 790 ff., JuS 1981, S. g6 ff., 409 ff., 488 ff.; M. Stolleis, Rechtsordnung und Justrypolirik 1945-1949, in: Europäisches Rechisdenken in Geschichze und Gegenwan, Festschrif f. H. Caing, Bd. I, Munchen I $982,5.383$ ff. 
polemisch verfehle, zu der näheren Bestimmung dieses Begriffs. In der Tat muß er, zumal für die Ausformung der Rechrslehre, präziser gefaß̧ werden.

Restauration im klassischen Sinne, crwa die Restauration monarchischer Strukturen unter den Stuarts in England in der zweiten Hälfte des 17. Jahrhunderts oder dic Metternichsche Restauration legitimistischer Ordnungsprinzipien in der ersten Hälfte des 19. Jalurhunderts, meint die Wiederherstellung des gesellschaftlichen, politischen und verfassungsrechrlichen Systems, das durch eine demokrarische Gegenbewegung umgewälzt werden sollte.' Dieses Theorem läßt sich auf den Entwicklungsprozeß nach 1945 nicht unmitcelbar, sondern nur in differenzierter Weise beziehen. Denn "Restauration « kann nicht die Rekonstruktion der politischen und rechtlichen Herrschafusbezichungen des NS-Systems bedcuten. Institutionell wird ja eine rechrs- und sozialstaatlich veríaße Demokratie geschaffen. Die konkrete Geltungsform der neuen Rechtsordnung wird jedoch in den Bereichen, in denen das Weiterbestehen der bürgerlichen Gesellschaft und ihres Staatsapparats gefährdet erschcint, einem rückwärtsgewandten Vorbehalt unterstellt. Dies geschiehr durch die weitgehende - allerdings durch das Bundesverfassungsgeriche der joer Jahre auch eingeschränkte - Interpretationsmachr der konservativen juristischen Funktionseliten, die zumeist die Diktatur des NS-Staates mitgetragen hatten. In der verdeckten und offenen Auseinandersetzung mir der $194 / 46$ von den Alliierten und deutschen politischen Kräften getragenen Tendenz, die Kontinuität des Systerns der NS-Diktazur und der durch sie gesicherten gesellschaftlichen Grundlagen mittels der Ausivechselung zentraler Trägerschichten des Dritcen Reiches zu unterbrechen, ${ }^{6}$ bilden sich bestimmte juristische Denkfiguren heraus, die sich zu einem restaurativen Rechtsbegriff zusammenfügen.? Wesentlich vier unterschiedliche, aber zusammenhängende Momente maclien dic Totalitär dieses restauraciven Rechrsbegriffs aus:

Zum einen werden Konstrukcionen entworfen, die zu verhindern suchen, daß es zu einer Diskontinuität zwischen dem Staatsapparat der NS-Dikratur und der neukonstituierten Demokratie kommr (I);

zum anderen werden Exkulpationslehren entwickelt, welche die Beteiligung bürger-

4 W. Conze, Stazs- und Nationalpolitik. Koncinuiciusbruch und Neubeginn, in: Soxialgeschichtc der Bundesrepublik Deueschland. Bcilräge zum Kontinuitassproblem, hrsp. von W. Conze und M. R. Lepsius, Stutgart 1983, S. 441 ff. (462 (.); differenxiener: J. Kocks. Restauration oder Neubeginn? Deutschland 1945-1949, L'76 H. I1/1979, S. 112 ff; R. Lowenthal, Dauer und Verwandlung, in: R. Lowenthal und H. P. Schwarz (Hg.). Die zweice Republik, Stutgarn 1974, S. g ff. (ro (.).

, Vgl. Th. Pirker, Die vetordnete Demokracie. Grundlagen und Erscheinungen der a Rescapration «, Berlin 1977, S. 268 \%

6 Zur Archäologie der Demokratie in Deueschland. Analysen amerikanischer Emigranecn im amenikanischen Gchcimdienst, Bd. 1: 1945-194s, hrsg. von A. Söllner, Frankfun a, M, 1982; D. Winkler, Die ancrikanische Sozialisicrungspolitik in Deutschland 1945-1948, in: Polivische Weichenstellungen im Nachkriegsdeutschland $1945^{-1953}$, hrsg. von H. A. Winkler, Geschiche und Gesellschaft Sonderhef, 5, Götungen 1979, S. 88 fl. (90 S.); Pousdamer Abkommen yom 2. August 1945 Abschnitu Ill, Kecsings Archiv der Gegenwart 1945, S. 14 f.; Minifest der demokracischen Sozialisten des ehemaligen Konzentracionslagers Buchenwald vorn 13. April 1945, abgedruck in: W. Abendroth, Aulsrieg und Krise der deutschen Sozialdemokracic, Fraskfun a. M. 1964, S. 123 ff.; Aufruí des Zentralkomitees der XPD vom 11.Jun 1945 an das deutsche Volk zum Aubau cins antifaschiseisch-demokratisclien Deurschlands. auszugsweise abgedruckı in: E. U. Huster, G. Kraiker, B. Scherer, F.-K. Schlotmann, M. Welieke, Decerminaneen der westdevischen Restauraxion, Frankfur 1972, S. $356 f f . ;$ V. Agariz, Sozialistische Wirschaftspolituk. Referat auf dem Parteitag der SPD in Hannover im Mai 1946, auszugsweise abgedruckt ubd.. S. 370 ff.; Das Ahlener Wirtschafısprogramm der CDU für NordrheinWestialen, ebd., S. $4 \geq 4$ ff.

7 Zum Restaurationsbegriff vgl, vor allem W. Dirks. Der restaurarive Charakter der Epoche, Frankfurter Hefic H. g/roso. S. 94 If,: E. Kogon, Beinahe mil dem Rucken zur Wand. Frankfurcer Hefice H. g/1954, S. 64I ff.: Th. W. Adorno, W/2s bedeutct: Aufarbeiung der Vergangenheit (1959), in: ders., Eingriffe, Frinkfurt 1. M. 1963 , S. 12 , II. 
licher Funktionsgruppen (Richtern und Ärzten) an der Terrorpraxis des NS-Sazates 361 in weirem Maße der Ahndung zu entziehen vermögen (II); sodann werden die in der Verfassung der Bundesrepublik fesigelegten rechtsstaatlichen, durch politische Freiheitsrechte gesichercen Begrenzungen der Staarsgewalt zur Erweiterung der Zugriff́smöglichkeiten auf den sinneren Feind « interprerativ gelockert (III);

paraliel dazu werden schließlich Argumentationsmuster eingeführt, durch die Verfügungen des demokratischen Gesetzgebers über die gesellschaftlichen Grundlagen - also die legale Überwindung der das NS-System fundierenden ökonomischen Ordnung - erschwert, jà tendenziell ausgeschlossen werden können (IV).

Für die Wiederherstellung der bürgerlichen Gesellschaft nach 1945 hatre der restaurative Rechrsbegriff insolern besondere Bedeutung, als er die Parrikularinteressen der an eincr weitgchenden Kontinuität interessierten Schichten von Besizz und Bildung, von Administration und Justiz methodisch in dic Form eines jeden verpflichtenden allgemeinen Rechts hüllte, das damic um so stärkere Legitimations- und Durchseczungskraft erzielen konnte. Die Entwicklung der Bundesrepublik wurde von den Formen dieses restaurativen Rechtsbegriffs nachhaltig geprägt; im ïberviegenden Teil der Lehre und der Rechtsprechung wirkt er bis heute weitcr. Daher ist die - notwendigerweise zunächst skizzenhafre - Aufarbeitung der Bestimmungsmomente des restaurativen Rechrsbegriffs keineswegs von antiquarischem Interesse.

Die Entwicklung des restaurativen Rechtsbegriffs wird an Hand einzelner theorerischer Leitfiguren der Rechcslehre exemplarisch nachgezeichner - ohne Anspruch auf eine auch nur in Ansärzen vollständige Analyse der juriscischen Disziplinen und Denkrichtungen. Die Repräsentanten der Rechtslehre erscheinen nicht als unmitzelbare, gar wböswilligex Subjekte des Restaurationsprozesses; sie sind gedankliche Träger realer geschichtlicher Tendenzen. Thre rechtstheoretischen Konstrukcionen werden auf das politische Strukturproblem staatliches und sozialer Neuordnung nach 1945 bezogen, das in den juristischen Interpretationen meist nicht direks sichtbar ist. Die Untcrsuchung beschränkt sich auf Tendenzen der Rechtslehre, auch wenn einige Hinweise auf deren Auswirkungen auf die Rechtsprechung gegeben werden.

\section{Legitimation der Rekonstruktion des alten Staatsappayats}

Die von den Alliierten nach 1945 intendierte Zerschlagung des NS-Systems vollzog sich in einem zusammenhängenden Bündel von Rechrskonstruktionen. Angeleitet durch zwei grundlegende, in der führenden juristischen Zeitschrife der USA veröffentlichte Aufsätze von Hans Kelsen, den die Nazis ins Exil getrieben hatten, wurde davon ausgegangen, daß die Niederwerfung des Nacionalsozialismus nicht bloß zu einer Besetzung eines im übrigen intakı gebliebenen Staaces geführt habe. ${ }^{8}$ Durch die - entsprechend der Berliner Deklaration vom s.Juni $1945^{9}$ - von den Alliierten ausdrücklich übernommene oberste Staatsgewait sei die Folge eingetreten, daß das Deursche Reich als Staat untergegangen und an dessen Stelle ein Kondominium der

8 H. Kelsen, The Intenational Legal Status of Germany To Be Established Immediately upon Termination of the War, American Journal of Law. Bd. 38 (1945), S. 689 ff.; ders., The Legal Stalus of Germany According to the Declaration of Bcrin, cbd. Bd. 39 (1945), S. 518 ff.

9 Kecsings Archiv der Gegenwart 1945. S. 257. 
Alliierten getreten sei. In der Tat bestand zwischen einer militärischen Besetzung, welche - der Haager Landkriegsordnung folgend - die Institutionen des besiegten Staates prinzipiell bestehen läßr, und der Übernahme sämtlicher staatlicher Funktionen - der legislativen, exekutiven und judikariven-durch die Siegermächce von 1945 eine unabweisbare Differenz. So war es kein Zufall, daß die Interpretation Kelsens in der Völkerrechtslehre 1945/46 und im Selbstverständnis der Alliierten weithin Anklang fand, ${ }^{\circ}$ weil sie das neue Phänomen einer über die milirärisclıe Niederkämpfung hinausgehenden Totalübernahme und Neukonstitution der bisherigen Staatsgewalt theorecisch zutreffend erfaßte. Kelsen selbst brachte die mit dieser juristischen Deutung verbundene politische Intention der Alijierten äuRerst klarsichtig im Singe einer vollständigen organisatorischen Diskoncinuität zwischen der Nazi-Despotie und dem neu zu gründenden demokratischen deutschen Staat zum Ausdruck:

- Nachdern die Periode des Kondominiums beendet isc und die Souveränirär Deutschiands wiederhergestell ist, wäre Deutschland rechelich ein neuer Staat. Es bestünde kcinc Kontinuizär zwischen dem zerstörten Nazi-Staat und dem neuen demokratischen Deutschland. Die neue Konstituion des souveränen Deutschlands wäre nicht das Resultat eines konstitutionellen Wandels innerhalb der Nazi-Verfassung, sondem der Beginn eines neucn Verfassungslebens. Nur als ein Gemeinwesen, das durch keine lcgalen Verbindungen mir Nazi-Deuschland verknüpft isı, sollte das demokratische Deutschland in die internationalen Organisationen nach diesern Krieg eintreten. ${ }^{11}$

Durch die Konstruktion des Untergangs des deutschen NS-Reichs waren unmittelbar die Träger des alten Staatsapparars betroffen: Indem das Rechessubjekt Deutsches Reich wegfiel, an das die Beamtenschaft juristisch gebunden war, ${ }^{12}$ wurde dic Kontinuität des überkommenen Staatsapparats unwiderruflich unterbrochen. Dem entsprach die Rechtspraxis der Alliierten und der von ihnen sich herleitenden Geserzgebung der Länder. Führende Beamte der NS-Diktacur wurden aus ihren Stcllungen endgültig enclassen: zunächst etwa $\$ 3000$ Personen. ${ }^{13}$ So hieß es beispielsweise in einem Beschluß des Staatsministeriums von Württemberg-Baden vom 5. Dezember 1946, der die Kontrollratsdirektive Nr. 24 vom I2. Januar $1946^{14}$ ausführte:

„Beamce, die durch die Militz̈rregierung, auf Befehl der Milizärtegicrung oder sonst aus politischen Gründen aus ihrer Stellung enfernt worden sind, getten nach der ausdrücklichen und verbindlichen Anweisung der Milicärregierung .... als entlassen mil der Folge des Verlusts ihrer sümtlichen Beamtenrechte. \&'s

Io R. Siödicr, Deueschlands Recheslage, Hamburg 1948, S. 36 ff., S. $\{2$ Anm. 42 m. w. Nachw., In Deutschland wurde diese Interpretation aufgegrifllen von J. v, Kempski, Deutschland als Völkerrechusproblem, Merkur 1947, S. 188 ff.; W. Abendroth, Dic Haltung des Rciches. Preußens, der Mark Brandenburg und der Cebieuskorperschafren des oflentlicheo Rechts für Verbindlichkeiten, die vor der Kapitulation vom 8. 5. 1945 intstanden sind, Neuc Juseiz H. 4/5/1947, S. 73 ff.; H. Nawiasky, Dic Grundgedanken des Grundgeretzes für die Bundesrepublik Deutschland, Stuttgan 1950, S. 7 ff.; vgl. auch dic Diskussionsbeiträgc von Abcnúloth und Nawizsky aul der Staarsrecheslehrenagung von r954 zum Thema: Der deutsche Staze im Jahre 1943 und seithcr, VVDStRL is (1955), S. 59 ff, S. 64 ff

1) H. Kelsen, The International Logal Status of Germany, 2. 1. O., (Anm. 8), S. 693. Hervorhebung von Kelsen. Zur Position van Kelsen vgl. auch die detailliente Darstellung von B. Distelkamp, a. a. O. (Anm. 3, JuS 1980), S. 481 ff.

12 Vgl. U. Scheuncr, Dic staalsrecheliche Kontinutäl in Deutschland, DÖV 1990, S. 481 ff. Von der Gegenposition ausgehend sprach Scheurer davon, xdab das Rrich als Dienstherr der Reichsbeamren erhaleen geblieben isto (S. $4^{84}$ ).

1) L. Nicthammer, Zum Verhälnis von Reform und Rekonstruktion in der US-Zone am Beispicl der Nevordnung des öffentlichen Dienstes, in: W. D. Narr und D. Thranharde (Hg.), Die Bundesrepublik Deutschland. Entstehung. Entwicklung, Srruktur, Konigstein 1979, S. 47 If. (52). Die Zahl beziche sich auf dic Weszonen.

14 Amtrblatl des Konerolliras 1946 Nr. S, S. 30.

is Zit, nach W. Jellinek, Bcamenrecheliche Ansprüche crelassener Beamier un der amerikanischen Zone nach recheskräfig abgeschlossenem Sprach kammerverlahren, DÖV 1949, S. 67. 
Über die völkerrechrliche Diskontinuitätstheorie und ihre innerstaatliche Durchser-

zung hinaus volizog die alliierte Gesetzgebung den rechtlichen und politischen Bruch mit dem NS-Staat ncben der Aburteilung der Kriegsverbrecher vor allem damit, die terroristische Staatspraxis im Inneren wegen der systematischen Verletzung menschenrechelicher Kerngarancien zu ahnden. Das Ziel war, die Exekutoren despotischer Staatlichkeit nicht bloß politisch auszuschalren, sondem mic ihrer Verurteilung zivilisarorische Mindeststandards als rechtlich unaufhebbar festzulialcen. Als Instrument hierfür fungiene das Kontrollratsgesetz Nr. 10 vom 20. 12.1945 $5^{16}$ mit dem Tarbestand der Verbrechen gegen die Menschlichkeir (Art. II Ziff. rc). Sie wurden definiert als "Gewalttaten und Vergehen ...: Mord, Ausrottung, Versklavung, Zwangsverschleppung, Freiheitsberaubung, Folterung, Vergewaltigung oder andere an der Zivilbevölkerung begangene unmenschliche Handlungen; Verfolgung aus politischen, rassischen oder religiösen Gründen, olıne Rücksicht darauf, ob sie das nationale Recht des Landes, in welchem die Handlung begangen worden ist, verleczen. $\alpha$

Die Zielsetzung der Alliierten, die Berufung auf das nationalsozialistische Rechtssystem abzuschneiden, weil es im Kern - mit den Worten von Franz Neumann nichts anderes war als eine Technik zur Manipulienung der Massen durch Terror "', fand auf deutscher Seite ihre Entsprechung in Gustav Radbruchs 1946 entwickeltem Theorem vom «gesetzlichen Unrecht *. Radbruch schrieb:

*Wenn Geserze ... Menschenrechte ... nach Wilkür gewähren und versagen, dann fehlt diesen Gesetzen die Geltung, dann schulder das Volk ihnen keinen Gehorsam, dann müssen auch Juristen den Mut finden, ihnen den Rechtscharakter abzusprechen. $u^{18}$

Radbruch gab ähnlich wie die Aufzählung im Kontrollratsgesetz Nr. 10 Beispiele für geserzliches Unrechc:

nWenn die Ermordung politischer Gegner gechrt, der Mord an Andersrassigen geboten... wird, so ist das weder Gerechtigkeit noch Recht. " Der Rechtscharakter fehlt ... allen jenen Gesetzen, die Menschen als Untermenschen behandelten. ${ }^{19}$

Die Konstruktion der * Verbrechen gegen die Menschlichkeit « und des »gesetzlichen Unreches « entzog dem maßnahmestaatlichen NS-Recht dadurch radikal die Legirimationsgrundlage, daß dessen rechtlich bindende Qualität nicht anerkannt und so die Geltung der nazistischen Unterdrückungs- und Diskriminierungsnormen von Anbeginn außer Kraft gesetzt wurde. Damit war der Weg für eine juristische Aufarbeitung staadich organisierter Willkür geölfnet.

Etwa Ende 1946 aber begann sich die politische Gesamtsituation in Deutschland im Zuge des Kalten Krieges zu ändern. Die von den USA dominierte Politik der Westalliierten tendierte immer stärker in Richtung auf die Restabilisierung des privaten

16 Amisblatt des Kontrollrats 1946 Nr. 3, S. 22.

17 F. Neumann, Behemoth. Surukeur und Prayis des Narionalsazialismus (1942/44), brsg. von Ger Schäfer, Koin 1977. S. s30.

18 G. Radbruch, Rechusphilosophische Besinnung (1243), in: ders., Der Mensch im Recht, 2, Auf?. Göttingen 1961, S. 106 .

19 Ebd.; G. Radbruch, Geseczliches Unrecht und übergesetzliches Rechr (1946), a. a. O., S. 120. Die Konsequenzen, dic für Radbruch aus dieser Grundposition folgtcn, sind freilich ambivalent: Richter, die geseizliches Unrccht grak wizierten, begingen zwar mobjekciv Rechrbeugung* (S. 123). Ihren Vorsauz zur Rechubeugung zog Radbruch aber in Frageform in Zweifel: . . . Konnecn Richter. die von dem herrschenden Positivismus soweit verbildet waren, daß sie ein anderes als das geserzle Reche nicht kannten, bei der Anwendung posiuver Geseize den Vorsac der Rechusbeugung haben? a (ebd.). Allerdings blieb Radbruch hierbei niche seehen: Rechiblindheil gegenüber gesetzlichem Unreche schloß für ihn den Vorsatz niche aus: Anm. zu OLG Frankfure a. M., SJZ 1947, S. 634. Vgl. hierzu I. Muller, Die Verwendung des Rechesbeugungstacbessands zu polirischen Zwecken, KJ H. 2/rg84, S. 120 f. 
Wirtschaftssystems ${ }^{20}$ und der - besonders von Frakcionen der amerikanischen Industrie von 1947 an propagierten - schritrweisen Beendigung der entnazifizierenden Ausschaltung der ökonomischen und administraciven Oberschichten, " auch wenn die anglo-amerikanischen Besatzungsmächte am Programm einer - schließlich gescheiterten - demokratischen Strukturreform des öffenclichen Dienstes noch länger festhielsen. "2 So bekamen die Interessen der einstigen staaclichen Funktionsträger des Dritten Reiches im gesellschaftlichen Kräfrespiel wieder ein größeres Gewicht. ${ }^{212}$ Entsprechend wurde das rechtswissenschaftliche Denken in den Karegorien der fundamentalen Diskontinuität zwischen dem Staatsapparat der NS-Diktatur und der neu konstituierten Demokratie in wesendichen Bereichen in die Defensive gedrängt.

Für die Cliarakterisierung des Rechtssystems unter dem Nationalsozialismus wurde - übereinscimmend mit dem nun in den Vordergrund rückenden liberalkonservativen Flügel der amerikanischen Regierung" - die lcgicimatorische Generalthese entwickelt, daß sowohl die Justiz als auch die juristischen Interpretationseliten der NS-Diktatur keine Verantwortung für die terroriscische Struktur des Regimes trügen, sondern allein die engere politische Führung.

Repräsencativ für das herrschende Bewußtsein der damaligen Rechtswissenschaft verfocht Eberhard Sctumidt zusammen roit anderen Autoren auf dem Godesberger Juristentag von 1947 diese These. Mit starker Betonung erklärte er - der Satz ist in der Druckfassung kursiv gesetzi -:

"... Nichr die Justiz, sondern ganz allein der Gesetzgeber halce die Fahne des Rechts verlassen. Und mit der Veranı wortung für die Folgen dürfen heute weder Rechtslehre noch Justiz bcladen werden, da dicse ganz allein den um jeden rechrlichen Halt gekommenen Gesetzgeber triffe. $x^{24}$

In die gleiche Richtung zielte die - nach dem Tagungsprotokoli ausdrücklich mit Beifall aufgenommenc - Bemerkung des Nordrhein-Wesdälischen Justizminissers Sträter auf dem Konstanzer Juristentag von 1947:

- Es darf nicht vergessen werden, daß ein großer Teil der Richer cine schwere Aufgabe erfüllt hat, er hat den Versuch gemacht, das Recht gegen dic Gewalthaber zu schützen. In seiner überwiegenden Mehrheit hat der deussche Richter vor Hider nicht kapituliert. ${ }^{21}$

20 E. Schmidr, Die verhindere Neuordnung, Frankfurr a. M. 1970, S. 1s0if; D. WinkJar, 2. 2 O. (Anm. 6) macht auf wichtige Gegentendenzen innerhalb der amcrikanischen Regierung - vor allem im Außenministerium - aufmerksam, die sich allerdings gegenüber der prokapitalistischen Politik des Milicargouverneurs der USA, Lucius D. Clay, niche durchzuserzen vermochen.

21 L. Nierhammer, Dic Mitläuferfabrik. Dis Entnazifizierung am Beispicl Bayerns, 2. Aufl, Berlin 1982. S. 486 If.

22 W. Benz, Versuche zur Reform des öffentichen Dienstes in Deutschlnd 1945-1952, Vieneljahreshefic: for Zeitgeschichee H. $2 / 9981$, S. 236 ff.

22a Vgl. dic cindringliche, zeingenossische Schilderung dieses Prozesses durch den seit 1946 im hessischen Justizministerium tötigen sozialdemokratischen Ministerialdirektor K. Oppler, in: H. Grebing (Hg.), Lchrstücks in Solidarität, Briele und Biographien deutscher Soziatisten 1945-1949, Stuztgar 1983, S. 290 ff.

23 L. Nicthannmer, a. 2. O. (Anm. 21), S. 34 ff., S. 490.

24 E. Schmidt, Unabhängigkcit der Rechtspflege, in: Tagung Deutscher Juristen Bad Godesberg 30. September/r. Oklober 1947. Reden und Vorríge, Hamburg 1947, S. 231; ebenso: E. Nierhammer, Der Kampf um die Wahrheit im Strafverfahren, a. a. O., S. 196: „Die weil überwicgende Melsrzahl der deutschen Richter versah ..., ohne sich vom rechten Weg ablenken zu lassen, das richcerliche Amt auch fernerhin pflichtbewußt und treu im reinen Streben nach Wahrheit und Gerechtigkeit. Das traf auch auf Ruchuer zu, die in besonderen Gerichten uber verbrecherische staitsfeindliche Betätigungen oder stazesgefahrliche Bestrebungen zu ureilen hanen.w E. Schmidt behuelt seine unkritische Bewertung der Rolle der Justiz im Dritcen Reich im wexentichen bei: E. Schmide, Lchrkommentar zur StPO und zum GVG, 2. Aufl. Göuningen 1964, Rdnr. 494 Anm. 329

2) Sträter, Diskussionsbeitrag, in: Der Konstanzer Juristentag, 2.-s. Juni 1947, Tübingen 1947, S. I8 I. 
Was für die Jusciz unterstellt wurde, das galt aus dieser Sicht für den gesamten Beamtenapparat des Dritten Reiches. In einer schon 1946 weirgehend abgeschlossenen, aber erst 1949 veröffentlichten Schrift behauptete einer der führenden konservativen Beamtenrechtler, Carl Heyland, daß die Beamten unabhängig von der jeweils geltenden demokratischen oder diktatorischen Verfassung allein der sogenannien ¿Staatsidee* gedient häıten. Derart wurde ihre Rollc in NS-Staat unangreifbar, weil sie mit der politischen Diktatur vorgeblich nichts zu tun hatten:

"Das Aufgehen in der über den Pareien stehenden Staztsidee hat dem deutschen Beamten die Kraft verliehen, unter jedem in Deutschland herrschenden politischen System, in der absoluten und konstitutioncllen Monarchie, iл der demokracisch-parlamentarischen Republik wie im nacionalsozialistischen Führerstaat in vorbildlicher Phichterfüllung und Sclbstlosigkeit zu dienen. $4^{26}$

Wenn man sich die Entfernung von Sozialdemokraten, Freimaurern, Liberalen und Juden aus dem Staatsdienst, die exzeßhafte Anwendung der Hochverratsbestimmungen gegen dic politische Opposition, die Durchsetzung der Repressionsnormen des NS-Arbeitsrechts und die Instrumentienung der gegen die Juden gerichteten Diskriminierungsgesetze vor Augen führt, ${ }^{27}$ wird deuclich, daß Beamtenschaft, Justiz und die ihr interprevierend zuarbeitende Rechtswissenschaft ein wesentlicher Faktor für die Aufrechterhaltung der nationalsozialistischen Herrschaftsbezichungen waren. Aus dem eben diese Realität verleugnenden ideologischen Selbstbild der juriscischen Trägerschichten des Dritzen Reiches ergab sich aber konsequent, die judikativen Institurionen und die juristischen Interpretationseliten, die mit dem naziscischen Terrorzusammenhang ja niches zu tun gehabt hätten, auch unversehrt bestehen zu lassen.

Der legitimatorischen Abrrennung des Staztsapparats von der NS-Führung korrespondierren juristische Theorien, die nun positiv eine Brücke für die Begründung der Kontinuität der staatichen Funktionsträger des Dritten Reiches konstruierten. Gegen die 1945 noch dominierende These vom Uncergang des Deutschen Reiches als Rechtssubjekt bildete sich parallel zur Umorientierung der westalliierten Deutschlandpolitik eine starke Gegenbewegung heraus, in der die bis heute fast unangefochten herrschende Identitätstheorie entwickelt wurde. Diese Theorie, die $194 \delta$ wesent lich von Rolf Stödcer, aber auch von Wilhelm Grewe und Erich Kaufmann ausgearbeizet wurde, ${ }^{28}$ hatte ihren Kern darin, die Existenz des deutschen Staates im werhisch-politischen Sinne" zu behaupten, unabhängig von einer ihn auch tragenden Rechrsondnung. ${ }^{29}$ Angesichts dieses vornormativen, metaphysischen Staatsbegriffs konnce die Übernahme sämclicher staaclicher Funkcionen und der Rechrsordnung

26 C. Heyland, Das Berufsbeamtentum im ncuen demokracischen Seaat, Berlin 1949, S. 33. Diese Siche der Rolle der Beamten um Driuten Reich fand sich zu Beginn der socr Jahre auch beim Bundesgerichushof: s. BGHZ 13, S. 196: „Das Bcamentum [hat]... Funtrionen, die der Staal als solcher immer übe und die weitgehend unabhängig sind von seiner wechselnden Erscheinungsform und den in diesem Wechsel sich ausdrückenden politischen Kraiten*.

27 Gesetz zur Wiederherstellung des Berulsbeamtentums vom 7. Aprl 1933. RGBI I, S. 175: Purisius, Reichssurafgesetzbuch. Leipziger Kommentar, 6. Aufl. Berlin 1944, S. 376; Kohlrausch-Langc, Stralgeseizbuch mit Nebengesetzen und Erliuurerungen, 37. Aufl. Berlin 1941, S. 242 Anm. 4i Th. Blanke, R. End, U. Mückenberger, U. Stascheit (Hg.), Kollektives Arbeitsrecht. Quellentexte zur Geschichte des Arbeitsrechts in Deutschland Bd. 2, Hamburg 1979 , S. 95 H.; R. W.alsner, Faschismus und Arbcitsrecht, in: U. Reifner (Hg.), Das Recht des Unrechisscaacs. Frankfurt 1981, S. 86 ff.; H. Robinsohn, Justiz als politische Verfolgung. Die Rechtsprechung in $*$ Rasseschandefällena beim Landgericht Hamburg 1936-1943. Stungart 1977; A. v. Brünneck, Dic Justiz im deveschen Faschismus, in: Redaktion Kritische Jusciz (Hg.), Der Unrechls-Scaut 1, 2. Aufl. 1983 . S. 108 II.

28 R. Stodicr, 2.2. O. (Anm. 10); W. Grewe, Ein Besatzungsstatue fur Deueschiand, Scutcear 1948; E. Kaufmann, Deutschlands Rechtslagc unter der Besaizung. Srutigan 1948. Zur Formierung der [den(itätstheoric vg). cingehend B. Distclkamp, x.2. O. (Anm. 3, JuS 1980), S. 48, f.

29 R. Stödicr, a. a. O., S. $4^{2}$. 
durch die Alliierten nicht zu Buche schlagen. Stödter scheute sich nicht, seinen außerrechtlichen Staarsbegriff, durch den zumal die demokratische Rechtsordnung als Konstituens des Staates außer Betracht bleibr, u. a. durch den Rückgriff auf einen führenden nationalsozialistischen Verfassungsrechtler zu begründen. Er rekurrierce auf eine Formulierung von Otto Koellreutter, demzufolge ein Staat so lange besteht,

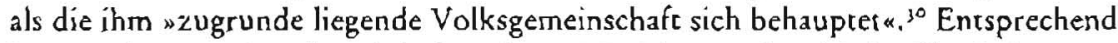
inrerpretierte Stödter die Niederlage des NS-Reiches zu einer bloß militärischen um, die politisch folgenlos sei: „Nicht das Deursche Reich, sondern die deutsche Wehrmacht hat im Mai 1945 bedingungslos kapiruliert. «" Die Übernahme der exekutiven, legislativen und judikativen Gewalı durch die Alliiercen verwandeles sich so in eine rechtlich bedeutungslose Äußerlichkeit. Die Fikcion einer weiterbescehenden, auf Volk und Reich gegründeten deurschen Staatsgewalt spitzre Stödter zu der These zu, daß das deursche Volk - entgegen der Realizät alliierter Souveränizär - die Stazrsgewalı weiter innehabe, jedoch akıuell nicht ausüben könne. ${ }^{32}$ Mit dieser Vorstellung wurde die Neukonstiturion der gesamten rechtlichen Ordnung des deutschen Staates durch dic Alliiercen ihrer einschneidenden Wirksamkeit beraubu. Die Folge war, daß die rechtlichen Beziehungen zwischen dem deutschen Staat als Rechtssubjekt und seinen personellen Trägern 1945 niche unterbrochen waren." So konnte die Fortexistenz des von den Narionalsozialisten strukcurierten Staatsapparats legirimiert werden - mit der bemerkenswerten zusätzlichen Konsequenz, daß die Säuberung des öffentlichen Dienstes von Republikanern durch die Regierung Hicler de facto mir sanktioniert wurde.

Unter den, Vätern des Grundgesetzes vertrat noch eine Minderheit die These, daß der deursche Scaat 1945 als Rechtssubjekt untergegangen sei. »... Auf dem Herrenchiemseer Konvent haben sich «, berichtete Carlo Schmid zusammenfassend, weine Reihe von Ländern auf den Standpunkt gestellt: Es gibt keinen deutschen Staat mehr, er muß neu konscituiert werden, und zwar durch Verräge der Länder. $\alpha^{34}$ Die Mehrheit des Herrenchiemseer Verfassungskonvents und des Parlamentarischen Rates ging jedoch von der Ansicht aus, daß der deutsche Staat über 1945 hinaus existiere und lediglich reorganisiert werden müsse. Wie weit dabei dic implizite Stoßrichtung der Identitätstheorie, den alten Staatsapparat rechtlich weiterbestehen zu lassen, von führenden sozialdemokracischen Verfassungsjuristen wie Carlo Schmid carsächlich erkannt worden ist, mag bezweifelt werden." Daß aber gerade diese Stoßrichrung hincer der Identicätstheorie scand', konnce an dem Affekt abgelesen werden, mit dem ein Vertreter der Identitätstheorie, Günter Krauss, 1954 die Anhänger der Diskontinuitä̈stheorie ausgrenzte:

30 A. a. O., S. ${ }_{4}$ 6. Zur Position von Ouo Kocllseutter vgl. ders, Vom Sinn und Wesen der nationalen Revolucion, Tübingen 1933.

31 R. Seödter, a. a. O., S. \2. Daßi sich die politischen und rechelichen Konsequenzen der Kapirulacion vom 8. Maj 194 sich nicht lediglich auf die mulitärische Führung Deulschlands bezogen, ging aus der Berliner Erflärung der Alliierten vom 1. Juni 1945 cindeutig hervor: „Die vier alliierten Regierungen übernehmeo hiermit in allen Deutsebland betreflenden Angelegenheieen dic oberste Autoric̈t, einschließlich aller Machrvolytomoenheiten, dic der deueschen Regierung, dem Oberkommando der Wehrmache und allen staadichen, städtisclien oder ördichen Regierungen oder Behärden zustehen.e Kecsings Archiv der Gegenwarn 1945, S. 257.

32 R. Stödter, a. 3. O., S. 48 .

33 U. Scheuner, 2. A. O. (Anm. 12).

34 C. Schmid, Rede anläßlich des is. Jahresıages der Verkundigung des Grundgeserzes, Das Parlament v. 10. Juni 1964, zit, nach W. Euchner, Der permanente Selbrbecrug. Zur Deuøschlandpolieik der Bundesregierung, in: C. Nedelmann und G. Schä(er (Hg.), Polistk ohne Vernunft, Reinbek 1965. S. 33; vgl. auch $H$. Nawiasky, 2. 3, O. (Anm. 10), 5. 101.

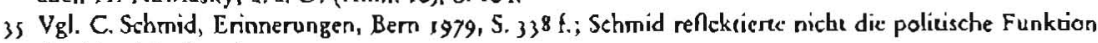
der Idenviätschcorie. 
"Dic deursche Staatsrechrlebre [hat] den Fortbestand des deurschen Staares mir cincr An moralischen Einstimmigkeit verteidigt $*$ w Staatsrechtslehrer, die sich... nicht in Deutschland befanden, . . . können in der Frage der moralischen Einstimmigkeit der deutschen Staatsrechtslehre nicht mitgezälylt werden $\alpha . .^{6}$

Diese Form der wwissenschafclichen « Ausbürgerung zielre vor allem au! den in den USA lebenden Hans Kelsen, der den rechtlichen Bruch zwischen der Staatsgewalt der NS-Despocie und der neu zu gründenden Demokracie unverrückbar zu markieren versuchte. Das politische Kernmoriv der Identitätstheorie trat damit unverhüllt ans Licht.

Im notwendigen Zusammentang mit der sich nun durchsetzenden Idencitätstheorie wurde die 194/46 erfolgre Entlassung erheblicher Teilgruppen des Beamtencorps der NS-Dikıacur in eine bloß zeirweilige Suspension umgedeuret, die den Kern des Beamtenverhälenisses unangecastet gelassen habe. ${ }^{7}$ Die sogenannte Suspensionstheorie - im Eigeninteresse der Beamten entwickelt - schloß direkt an die Identirätstheorie an. Die Maßnahmen der Alliierten gegen die Beamten wurden an der allein für eine Besetzung, nicht aber für die Übernahme des staatlichen Ordnungsgefüges geltenden Haager Landkricgsordnung gemessen, derzufolge lediglich Amtssuspensionen, keinesfalls aber die echce Entlassung nicht genehmer Beamten des okkupierten Staates gestatter sind. ${ }^{18}$ Haltbar wäre dieses Argument nur, wenn die Alliierten, anders als in ihrer Erklärung vom s. Juni 1945 über die Übernahme der sobersicn Autoritäı «" die deursche Staarsgewalt prinzipiell bestehen gelassen hätten. Angetrieben aber durch die der eindeutigen Praxis der Alliierten entgegengesetzte politische Option, daß es zur Ausschaltung des nationalsozialistischen Einflusses nur Enclassungen im tatsächlichen Sinne bedürfe, nicht aber der Aberkennung der Ansprüche aus dem Beamtenverhähtnis, ${ }^{\circ}$ wurde der Wortlaut der Kontrollratsdirekcive Nr. 24 vom I 2. I. 1946-»Personen, die aus öffentlichen Ämtern entfernc werden, haben kcinen Anspruch auf Ruhegehälter oder andere Beamten-

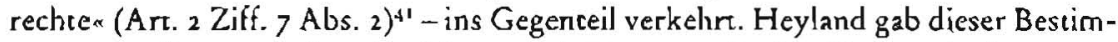
mung eine ihre Intention durchkreuzende Fassung, die den Verlust der Beamtenrechte befristete: "Personen, die aus öffentlichen Ämtern cntfernt werden, werden während ihrer Entfernung keinen Anspruch auf Ruhegehälıer oder andere Beamrenrechce haben. $x^{42}$

Gestützr durch die Suspensionstheorie konnte in der auslaufenden Entnazifizierung, die nach einem Wort von Franz Neumann tendenziell in eine "Renazifizierung " ${ }^{43}$ mündere, die Wiedercinscellung der einst entlassenen Beamten durchgesetzt werden:

36 G. Kmuss, Die Verfassung Deutschlands 1945-1954, DÖV 1954, S. \$\$0. Anm. 10. Weniger grob, aber mit einer ábnlichen Ziclrichtung bezog Hans Peter lpsen in einer knappen Bemerkung gegen Kelsens Diskoncinuitätsthese Secllung: Stödter habe mit seinem Werk: Deutschlands Recheslage (Anm. 10) *cine deuesche politische Tat vollbrache $\alpha$ - als sei die Ansichr von Kelsen glcichsam oundeuesch. Ipsen fuhr for: ${ }_{n}$ Noch vor Erich Kaufmann und Wilherm Grewe hat cr [Stodier] die besatzungspolicisch wesent. liche und insbesondere in den Vereinigteo Statuco von Hans Kelsen verbreinete These vom Kapitulationsuntergang des Deurschen Reiches widerlegt.* H. P. Ipsen, Ein Hamburger Laben für Wiruschale und Wissenschast, Festscheift I. R. Stödter, Hamburg 1979, S. XV1.

37 Vgl. die Darstellung von M. Kirn, a. a. O. (Anm. 3).

18 C. Heyland. Dic Rechtsstellung der endemen, erfolgreich entrazifizierten Beamsen, DÖV 1950, S. $326 \%$.

39 S. 0. Anm. 9 .

$4^{\circ} \mathrm{N}$. Wahl, Beamtenrechtliche Ansprüche entlassener Beamter in der amerikaaischen Zone nach rechtskräfig abgeschlossenen Spruchkammorverfahren, DÖV 1949. S. 22.

4 S. o. Anm. I4.

42 C. Heyland, 2 a. O. (Anm. 38), S. 325.

43 F. Neumann, Milicärrcgierung und Wiederbelebung der Demokratie in Deutschland (1948), in: ders. Winschuar, Seaal, Demokratic, Aufsälzc 1930-1934. brsg. von A. Sollner, Frankfor a. M. 1978, S. 324 . 
Von 33000 in den Westzonen Enclassenen kehreen knapp 32000 in den öffentlichen Dienst zurück. ${ }^{14}$

Das Bundesverfassungsgericht, das in seiner personellen Zusammensetzung so gut wie keine Verbindung zu den juristischen Funktionseliten des Dritten Reiches besaß," nahm in den Beamtenentschcidungen der soer Jahre die Diskontinuitätstheorie, zu dor es freilich formell nichr Stellung bezog, der Sache nach auf: ${ }^{66} \mathrm{D}_{\mathbf{a}}$ die Beamrenschaft integraler Bestandreil der NS-Diktatur gewesen war, erklärte das Bundesverfassungsgericht deren Rechte mit dem 8. Mai 1945 für erloschen. ${ }^{47}$ Die praktische Wirkung dieser Encscheidungen war allerdings gering, da die Inkorporation des alten Beamtenapparats schon fast vollständig abgeschlossen war. Immerhin erschürterte das Bundesverfassungsgericht die Legitimationsgrundlagen der an die Identiätstheorie gebundenen restaurativen Beamtenrechtsdoktrin.

\section{Anerkennung von NS-Gewaltpraxis als Reche}

Parallel zur legitimatorischen Rekonstruktion des alten, einst der NS-Diktatur dienenden Staatsapparats bilderen sich Argumentationslinien dafür aus, die Ahndung der Vcrbrechen dieses Staatsapparats zu beschränken. Verfolgen läßr sich dics an der Kritik an dem im Kontrollratsgeserz Nr. 10 kodifizierten Tatbestand des Verbrechens gegen die Menschlichkeit, der Ablehnung der Haftung von Richtern für ihre Tärigkeit im Dritten Reich und der Exkulpation der zu Tötungsgehilfen des Staates gewordenen "Euchanasier-Ärzte.

Unterstützt durch ein Gutachten von Eberhard Schmidt ${ }^{48}$ entwickelte der Präsident des OLG Celle, Hodo v. Hodenberg, 1947 eine Argumentation, durch die die Geltung des Kontrollratsgesetzes Nr. 10 mit der Begründung in Frage gestellt wurde, diese Norm, die ausdrücklich auch rückwirkende Geltung beansprucht, verstoße gegen den rechtssiaatlichen Grundsatz nulla poena sinc lege.

Diese Regelung führe zu einem "Rückfall $\alpha$ in das Rechtsdenken des Dritten Reiches:

×... An dic Stelle des den Richter bindenden Geserzes [wird] - genau wie in der Nazi-Zeir - das 'gesunde Rechisgefühl der Volksgemeinschafu gesezz. \& Damil werde »der Grundsaız der Nazi-Zeit « realisier, $\times$ daßs , der Schuız der Volksgemeinschaft den unbedingten Vorrang vor den Belangen des Individuums.... zu beanspruchen habe. ${ }^{49}$

Diese Argumentation richtete sich mindestens implizit auch gegen Radbruchs Theo$\mathrm{rcm}$ vorn gesetzlichen Unrechts das dic formelle technische (Un)Rechtsgelcung im nachhinein am Maßstab menschenrechtlícher Kerngarantien zu messen suchte.

44 L. Niethammer, a. a. O. (Anm. 1 j). Ein markantes Beispiel für die wiederhergesteller Konrinuitäi der staadichen Funktionseliten der NS.Diktatur war die Person von Hans Globke, Staatssekrezär im Bundeskanzleramt unter Konrad Adenauer, zuvor Minisecrialrat in Reichsinnenminisrerium und führend beteiligt an der juristischen Diskriminierung der Juden. Vgl. G. Petersen und N. Schirmacher, Dic Rekonstruktion des Beamtenapparats der Bundesrepublik Deutschland und die Rolle des Stantssekretärs im Bundeskanzlcramı Hans Globke, Magisterarbert, Univcrsicä́ Hannover 1984.

45 J. Fecst, 2, 2. O. (Anm. 1), S. 10, f.

46 Zutrclfend sicht dies in seiner Kritik an der Entscheidung des BVerIG: F. Forstholf, Das Bundesverfasungsgeriche und das Berufsbeamientum, DVBI. 1954, S. 69.

47 BVeriGE 3, S. s8 ff. und BVerfGE 6, S. I j2 ff. Zur Analyse s. auch M. Kirn, a. a. O. (Anm. 3).

t8 M. Broszat, Siegerjusuz oder strafrechuliche *Selbstreinigung $\alpha$. Aspekte der Vergangenheirsbewàllugung der deurschen Juseiz während der Besalzungszeit 1945-1949, Vieneljahreshefte for Z̈eirgeschichte, H. $4 / 1981$, S. 477 fl. (522). Broszat lieien eine decailiene Analyse der Auseinandersetzungen um das Kontrollratsgesecz Nr. 10 in der justiz der brivischen Zone (S. si6 ff.).

$49 \mathrm{H}$. v. Hodenberg, Zur Anwendung des Kontrollratsgesezzes Nr. so durch deulsche Gerichte, SJZ 1947. Sp. 120. 
Dabei verkannte v. Hodenberg, der die Auffassung weiter Teile der Justiz und der überwiegenden Mehrheit der Strafrechtslebrer repräsentierte, ${ }^{\circ}$ daß das Rückwirkungsverbot auf eine funkrionierende rechisstaatliche Ordnung bezogen ist," durch die die Individuen vor unberechenbaren Srrafansprüchen der öffentlichen Gewalt geschützt werden sollen. Das Schurzobjekt des Rückwirkungsverbors wurde vertauschc. Nicht mehr die autonomen Individuen, sondern die Träger terroristischer Staatsgewalt wurden sein Bezugspunkt. Damit aber veränderte das Rückwirkungsverbor seine liberale Aufgabenstellung. Es sicherte nicht melır die sraatsfreie Sphäre der Individuen, sondern die unangreilbare Geltung der Diskriminierungs-Legalität des nationalsozialistischen Staatsapparats. Indem das Rückwirkungsverbot ohne Einschränkung auf eine öffentliche Gewalt bezogen wurde, die sich selbst strukturell außerhalb rechisstaatlicher Schranken bewegte und selbsc den Grundsarz nulia роепа sine lege als Eingrenzung ihrer Scrafverfolgungsbefugnis verwarl, "2 verkehre sich seine rechtsstaatliche Funktion ins Gegenteil. Es wurde in den Bereichen, die das Kontrollratsgesetz Nr. 10 über die Strafdrohungen des deurschen StGB hinaus zu poenalisieren suchte, zum Garanten der sanktionsfreien Willkür-Staatsgewalt des NS-Systems. Die „Verfolgung aus politischen, rassischen oder religiösen Gründen « (Kontrollratsgesetz $\mathrm{Nr}$. 10), die etwa mir der Hochverratsjudikatur oder der Anwendung der Nürnberger Rassegeserze betrieben wurde, ${ }^{522}$ konnce der Ahndung entzogen werden. Die Schlußfolgerung lag auf der Hand: Nur wenn das Rückwirkungsverbor gegenüber einem terroristischen Staatsapparat, der es grundsätzlich mißachrete, für menschenrechtliche Kerngarantien formell durchbrochen wurde, konnte es seine eigentliche Funkrion, die öffentliche Gewalt an Strafexzessen gegenüber den Individuen zu hindern, auch wirklich erfüllen."

Die Grundposition, die Alindung von staaclich praktiziertem gesetzlichen Unrecht unter Berufung auf das Rückwirkungsverbor auszuschließen, bezog Helmut Coing in cinem richtungsweisenden Aufsatz von 1947 auf das Problem der Haftung von Richtern für die Anwendung naturrechtswidriger Gesecze. st Coing erklärte, da? Richter für eine Anwendung derartiger Geseıze nicht zur Rechenschaft gezogen werden dürften. Um eine scrafrechrliche Haftung für die Träger der NS-Justiz auszuschließen, berief sich Coing auf die Funktion des Sarzes mulla poena sine lege, die Macht des Gesetzgebers wim Interesse der Freiheit a" zu begrenzen - ohne zu bedenken, daß damic nicht etwa die Freiheit des autonomen Individuums, sondern die Willkürfreiheit des NS-Staatsapparats posiciv geschǚı wurde. Die nachträgliche Generallegitimation des Richters des NS-Staaces, dem zugute gehaiten wird, ndaß er in unmittelbare(m) Gehorsam gegen das Gesetz gehandelt hata, ${ }^{66}$ trat als Mociv von

so M. Broszat, a. 3. O. (Anm. 48), S. \{22.

1 V V.l. A. Arnde, Das Verbrechen der Euthanasic, in: Der Konscanzer Juristentag. a. a. O. (Anm. 21). S. 199

\$2 Gesetz zur Änderung des Stralgesetzbuchs vom 28. Juni r935, RGBI. 1, S. 839.

S2a S. Anm. 27.

53 Vgl. dazu auch G. Radbruch, Zur Diskussion über dic Verbrechen gegen die Menschlichkeil, SJZ 1947, Sp. 1) I ff. Radbruch [uhre den Gegoern des Kontrollratsgesclzes Nr. ro dic Konsequenz ibses Denkens eindrückJich vor Augen: - Wrill man emsettch die Folgerung ziehen, auch dic unter dem Namen Euchanasic verborgenen Ansialtsmorde deshalb straflos zu lassen, weil sie aut einem Geheimbefehi Hitlers beruheen...? ... Man würde dureh die Ablchnung, das Gesetz Nr, ro rickwirkend anzuwenden, den ganzen Sinn dieses Gesetzes mißdeuten und zerstören. * ( $S_{F}$. 1 34 ).

is $\mathrm{H}$. Coing, Zur Frage der straĺrechdichen Haftung der Richter lür die Anwendung naturrechtswidriger Gesecze, SJZ 1947, Sp. 6 r fí.

is A. a. O., Sp. 63.

s6 Ebd. Im Unterschied zu Coing blicb Radbruch nichr bei dieser Rechrferrigungschese stehen. Zwar metunte er, wder Positivismus mit scincr Übcrzeugung , Gesetz, ist Gesetzea babe nden deutschen Juristenstand wehrlos gemacht gegen Geserze willkürlichen und verbrecberischen Iohales a (Gesuczliches Unrecht, 2 2. O., Anm. 19. 5. 118). Zugleich aber entzog er einer Berulung aú das Terror-Recti des 
Coings Argumentation zu Tage: Die Anwendung der Hochverratsbestimmungen, bei der sich die rechtsstaatliche Schranken niederreißende, vielfach mörderische Qualizär der NS-Rechtspraxis besonders eindringlich zeigte, ${ }^{6 a}$ rechrfertigte er mit dcr Bemerkung, es sei »zu berücksichtigen, daß es nicht möglich ist, cine bestimmte Staarsform als absolut gerecht zu deduzieren ${ }^{17}$ Durch diesen unzulässigen Umkehrschluß und der damit ausgesprochenen Unterstellung, die NS-Justiz habe sich in Rahmen der allgemeinen und unvermeidlichen Abweichung vom Ideal bewegt, enzzog Coing einer juristisch folgenreichen Verwerfung der nacionalsozialistischen Hochverrarsrechesprechung den Boden. So wurde die spezifische Qualizät des politischen NS-Rechts, als Instrument des technischen Terrors gegen dic Opposition zu fungieren, zum Verschwinden gebracht..$^{88}$

Weniger subril als Coing, aber mit gleichem Ergebnis ging ein OLG-Rat aus Celle, Robert Figge, bereits 1947 zur direkren Rechtfertigung der NS-Justiz über. Er zog die nationalsozialistische Ideologie als positive Begründung für die Justizpraxis des Dritten Reiches heran. In einer bösen Umkehrung verwandelten sich damit die Verbrechen gegen die Menschlichkeit in Akte der Menschlichkeit:

"Der Richter, der den Nacionalsozialismus unterstützıe, konnze ... u. U. des Glaubens sein einer Hunıanität zu dienen... Solltc man es dem deutsclıen Richter wirklich verübeln, wenn er glaubre, in politisclien Prozessen einer guten Sache, einer höheren Humanität zu dienen ias"

Die Tendenz in der Rechtslehre, die Durchbrechung des menschenrechelichen Kernbereiclıs durch die NS-Dikcatur der Ahndung für unzugänglich zu erklären, konnte

NS-Regimes dic Grundlage: "... Von vornheren [war] auspesprochen, daß das nationalsozialisische Rechi sich der vesensbeszimmenden Anforderung der Gerechuigkeit, der gleichen Behaodlung des Gleichen, zu entziehen gewillt war. ... Infolgedessen entbehrte es... der Rechtsnatur, ist nicht etwa unrichniges Reclx, sondem uberhaupr kein Reche، (S. 120). - Freilich kann die These, daß der Posiuvismus und nicht etwa ihre autorstz̈ren Wertvorstellungen dic Richecr im NS-Regims swelsrlosgemache habe, längst nicht mchr aufrechterhaleen werden. Vgl. nur B. Rüthers, Dic unbegrsmzte Auslegung, 2. Aufl. Frankfun a. M. 1973; 1. Maus, Bürgerliche Rechlsheorie und Faschismus, München 1976, S. 37 If.; H. Wrobel, Die Anfechtung der Rassenmischehe, KJ H. 4/1983, S. 149 ff.; H. Ronleuthner, Substanticller Dezisionismus. Zur Funktion der Reclusphilosophie im Nacianalsozialismus, in: ders. (Hg.), Rechi, Rechisphilosophic und Nationalsozialismus, Archiv fur Reches- und Sozialphilosophie, Beihefit i8, Wiebaden 1983, S. 20 il.

s6a Parisius, a. a. O.; Kohlrausch-Lange, a- 4. O. (Anm. 27): R. Schmid, Einwände、Surgar 196s, S. 236 .

s7 Coing, a. a. O., Sp. 64. Politischen Diskriminierungen durch Verwaltungsaku des NS-Staatus hiclt Coing cbenfalls für rechisgülug. Allerdings fügre er einschränkend hinzu, daß derartige Maßnahmen nichr schlechthin für wirksam erkJär wcrden dürten, weil sonst Widerstand gegen sie omis ders Makel der Rechtswidrigkeil a belcg würde. OLG Sarbrücken DRZ 1947, m. Anm. v. H. Coing, S. 142 f., s. hierzu M. Swlleis, a. a. O. (Anm. 3), S. 404.

s8 Die Konsequenz dieses Denkens trat in der Rechesprechung des BGH zu Todesurteilen gegen Widerstandskämpfer zu Tage: Am 19. Juni 1956 sprach der BGH den Vorsizenden Richer,. Dr. Thorbeck der am 8. April 1945 im KZ-Flossenbürg das farcenhale Standgerichesvertahren u. a. gegen Dictrich Bonhoeffer gclcitec hatce, frci: = $A$ usgangspunks ist das Reche des Seasues au Selbsibchauptung. In cincm Kampl um Scin odor Nichusein sind bei allen Volkcrn von jeher strenge Gesetze zum Staatsschutze eriassen worden. Auch dem nacionalsozialistischen Staat kann man nicht ohne wciteres das Recht absprechen, daß er solche Gescuc crlassen hat ... Einem Richser, der damals einen Widerseandskämpfer wegen seiner Tätugkeit in der Widerstandsbewgung abzuurteilen hatte und ihn in einem einwandfreien Verfahren Jür uberführe erachtete, kann heutc in strafrechtlicher Hinsicht keis Vorwurf gemache werden, wenn er angesiches der Unterworfenheit unter die damaligen Gesetze nicht der Frage nachging, ob dem Widerstandskämpfer erwa der Recheferrigungsgrund des übergeseczlichen Notsunds unter dem Gesichtspunkt eines boheren, den Straídrohungen des stastlicben Gesetzes vorauslicgenden Widerstandsrechis zur Seite stehe, sandern glaubie, ihn des Hoch- und Landesverrats... schuldig erkennen und deswegen zum Tode verurteilen zu mussen.* Dic Entscheidung ist abgedrucke in: Justiz und NS-Verbrechen, Bd. XIIl, hrsg. von C. F. Rüler U. A., Amsterdam 1975, S. 144 If. (3) 2): in der amclichon Sammlung ist sic nicht enthaleen. Zur engehenden Analyse und Kritik der Rechtsprechung des BGH, derzufolge die Todesureile zegen anui-nazistische Widerstandskimpler rechelich prinzipiell gülug scien, s. G. Frankenberg und F. J. Müler, Jurisusche Vergangenheirsbewäleigung. Der Volksge-

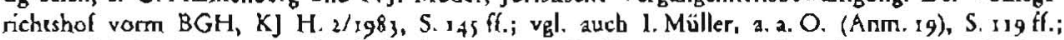
G. Spendel, Rechusbeugung durch Rechesprechung, Berlin 1984; zum Prozeß gegen Tharbeck s. H. Kraver, Filme zur NS-Juseiz, KJ H. y/r984, S. josf.

s9 R. Figge, Dic Verantworelichkeit des Richrers, SJZ 1947, Sp. 182. 
an der juristischen Bewertung der Praxis der in die staacliche Vernichrungsmaschinerie eingebundenen "Euthanasie«-Ärzte konkreter verfolgt werden. Waren zunächst "Euthanasie «-Ärzre, wesentlich gescützt auf Radbruchs Argumentation von der Unverbindlichkeit naturrechtswidriger, das Recht auf Leben beseitigender staatlicher Befehle, wegen ihrer Beteiligung am Anstaltsmord verurreilt worden, ${ }^{60}$ so bildece sich uncer der Führung von Hans Welzel und Eberhard Schmide wiederum eine Gegenströmung heraus, durch die die Euchanasie $\alpha$-Ärzte vom Vorwurf schuldhaften Handelns entlastet werden konnten.

Welzel baute jenen Ärzten, die am staarlichen Törungsmechanismus tejlhatcen, mit einer Ervägung eine goldene Brücke, die aus seiner schon damals voll encrickelten Irrtumslehre folgtc. ${ }^{61}$ Die Konstruktion, daß ein Täter bei einem unvermeidbaren Verbotsirrtum schuldlos gestelle werden kann, fand ihr soziales Substrat in der Exkulpation von Ärzten, die am Anstaltsmord beteiligt rwaren: Sie liäzten, argumenrierte Welzel, zwar carbestandsmäßig unò rechtswidrig, aber moglicherweise schuldlos gehandelt, weil sie subjekriv an die Rechtmäßigkeit des narionalsozialistischen Törungsbefehls glaubren. ${ }^{62}$ Zur Begründung griff Welzel auf die spärscholastische Moraltheologie zurück, die "für Euchanasie i. e. S. (die Sterbehilfe) die Möglichkeit eines unüberwindbaren, schuldlosen Verbotsirrtums anerkannt ‘ habe. Daher könne, entgegen der zunächst vom OLG Frankfurt a. M. entwickelten, auf Radbruchs Begriff des gesetzlichen Unrechts fußenden Rechtsprechung, in der nFrage der Zulässigkeit der Vernichtung lebensunwerten Lebens von einer ... naturrechrlichen Evidenz keine Rede sein. ( $^{\text {bs }}$ Mit dieser Argumentation ebnete Welzel die Fundamentaldifferenz zwischen einer individuellen Sterbehilfe, bei der der Sterbende als Subjekt respektiert wird, und der planmäßigen staatlichen Ermordung psychisch Kranker vollständig ein. Nur durch die apologerische Verzerrung des wahren Sachverlaalts des Anstaltsmordes war Weizel in der Lage, die Schuld der nEurhanasie«-Ärzte in Zweifel zu ziehen und dem unverbrüchlichen Schurz des Lcbens, wie er nicht zuletze durch die auch unter dem Nationalsozialismus fortgeltende Norm des $\$ 2$ I StGB zum Ausdruck kommt, die Evidenz zu bestreiten. ${ }^{6_{4}}$

Für den Fall, daß die „Euchanasie - Ärzte ihre Beteiligung an der Tötungsaltion damit recht\}́ertigten, sie hätten dies zu dem Zweck getan, einige Kranke zu retten, konstruierte Welzel gemeinsam mit Eberhard Schmidt einen übergesetzlichen Schuldausschließungsgrund. Welzel und Schmide kritisierten die Begründung ciner Entscheidung des Obersten Gerichrshofs der Britischen Zone, der diese Ärzte für schuldig erklärte, ihnen aber einen persönlichen Strafausschließungsgrund zubilligte. ${ }^{\text {os }}$ Weizel argumentierte: Die Ärzte hätten durch die Nichtbeteiligung an der Töcungsaktion und der damic verbundenen Unmöglichkeit, anderen Kranken das Leben zu erhalcen, eine größere Schuld auf sich geladen, als wenn sie an der Auslöschung psychisch Kranker mitwirkten, um einige der Tötungsmaschinerie zu entziehen. ${ }^{66}$ Eine derartige Überlegung konnte legitimerweise nur für die Strafzumes-

60 OLG Frankfurr 2. M. SJZ 1947, Sp. 633 m. Anm. v. G. Radbruch; KG DRZ r947. S. 198 m. Anm. von R. Lange.

6r H. Walzel, Der Irrum über die Rechrswidrigheit des Handelns, SJZ 1948, Sp. 368 If.

62 H. Welzcl, Arm. 24 OGH MDR 194\%, S. 375 .

63 A. а. O., S. 376; s. auch H. Welzel, Vom irrenden Getvissen, Tübingen J949.

64 Mit eirer der Argumentation Welzels cntsprechenden Begründung, bestimmten am Anstaltsmord beteiligten Ärzeen sci cine Schuld nicht nachzuwcisen, stellec z. B. das LG Hamburg 1949 ein Verfahren gegen is "Euchanasica-Ärzee ein. Der Spiegel Nr. $\$$ /1960, S. 2\}. Ebenso entschicd der BGH im Fall des nEuthanasica-Arzes Dr. Borm: KJ H. 4/1974, S. 428 ff. m. Anm. v. R. W Kempner, s. hierzu auch X. Blüchel, Das Mediyinsyndikal, Reinbek 1978, S. 73 if.

6s OGH SJZ 1949, Sp. 347 ff. (3ss f.).

$66 \mathrm{H}$. Welzcl, A. A. O. (Anm. 62), S. 375, ebenso H. Wclzel, Zum Noustandsproblem, ZSiW 63 (1950). S. 14 . 
sung, keinesfalls aber für die Bestimmung der Strafbarkeit vorgenommen werden. Der springende Punkt war, daß dic Ermordnung der nicht zu restenden Pacienten als "Wahl des kleineren Übeis zwar rechtswidrig blieb, aber durch den Ausschluß der Schuld im Ergebnis doch rechtlich zulässig wurde:

„Das Recht... kann den Täter nichı vor der Recbısgemeinschafı zur Verantwortung zielıen und ihn rechulich für schuldig erklären, sondern dic Schuld des Tälers muß das bleiben, was sie ist, eine Schuld vor dem Gewissen und vor Gott. $\alpha^{67}$

Indem Welzel die "Euthanasie "-Ärzte allein ssittich», nicht aber rechtlich für schuldig erklärte, vollzog er die maßnahmestaatliche Scruktur der Aufhebung des unverbrüchlichen, rechtlichen Schutzes des Lebens gedanklich nach.

Mit einer anderen Begründung kam Ebcrhard Schmidr zum selben Ergebnis wie Welzel. In einer grocesken Variarion der Identitätstheorie identifizierce Schmidt den Staat des Nationalsozialismus, der die Ermordung psychisch Kranker verfügre, mit dem neu konstituierten Rechusstaat, der die syscematische Durchbrechung des Schutzes des Lebens zu ahnden suchte:

* Der Staat, der es ferig bringl, den Armene [nämlich den -Euthanasic-Arzt, J. P.] sittlich schuldig verden zu lassen, hat jedes Recht verloren, ihm einen rechulichen Schuldvorwurf zu machen.. ${ }^{68}$

Durch ein metaphysisches Wunder blieb der von den Alliierten niedergekämpfre und zerschlagene Staat weiter existent und mußte sich ein strafrechtliches venire contra factum proprium vorhalten lassen.

Überblickr man dic bald nach Kriegsende sich ausbildenden rechrswissenschartlichen Grundströmungen der Identitärstheorie und der beamtenrechrlichen Suspensionstheorie, der Bewvegung gegen die Ahndung von Verbrechen gegen die Menschlichkeit und der Schuldlosstellung von "Euthanasies-Ärzten, so bildeten sic eine strategische Einheit: Sie arbeiteten der Unterbrechung der Kontinuität der Trägerschichten der NS-Diktatur und der radikalen juristischen Entlegitimierung der Rechrsordnung des Driteen Reiches enrgegen. Sozialpsychologisch betrachtet entsprang dies der Unfähigkeit, in rechtlichen Formen Trauerarbeic zu leisten. ${ }^{69}$ Die unverstellte und betroffen machende Wahrnehmung der vom nationalsozialistischen Staarsapparat ausgehenden Realicät des Terrors wurde durch eine Fülle von Abrvehrmechanismen, die die Gestalt juristischer Konstruktionen annahmen, unterbunden. ${ }^{\circ}$

Korrespondierend hicrzu unterwarfen Repräsentanten der alten Interpreracionseli-

67 H. Welzel, a. a. O. (Anm. 62), S. 375. In Welzels Aufsatz: Zum Nousiandsproblem (Anm. 66) hicB es cuispreclicnd: "Wo dic... sozialc: Vorwerfbarkeit fchl, weit jeder andere Rechesgenosse an Stelle des Tacers ebenso handeln mußte, fehle es auch an eincr Rechesschulda (5. 52). Diexe Konstruktion fubre im Blick auf die „Euthanasie -Ärzte, die sıch an der Tötung betciligzen, um einigen Paricnten das Leben zu ephalten, zu dem makabren Engebnis, daB - logisch zu Ende gedachs - die Nichuteilnahme am Anralcsmord einer $\triangle$ Rechesscluuld a gleichkame!

68 E. Sclumid, Anmerkung zu OGH SJZ 1949, Sp. 6/9. Schmide frage, oob man den Täter einer aus solcher Motivationslage entstandenen Tat [ramlich psychisch Kranke zu cöten, um einige zu resten. J. P.] als ,Verbrecher abstempeln darf. "Schmidt fuhr fort: „Der Stax hat hier niche Anlaß, einen - Verbrecher laufen zu lasscon, sondem er muß sich sagen lassen, daß er sich diesem Täter gegenuber mis seinen rechelichen Schuldvorwürten zurückzuhaltcn, da $B$ er ihn mangels Schuld niclit als Verbrecher zu werten hat. a (cbd.).

$69 \mathrm{Vgl}$. A. und M. Misscherlich, Dic Unfähigkcir zu erauem, Munchen 1977.

70 Mitulerweile wird dieser Prozeß offen gerechtfertigt. $V_{g l}$. H. Lubbe, Es Ist nichis vergessen, aber einiges ausgeheil. Der Nationalsozialismus im BewuBiscin der deueschen Gogenwart. Frankfurter Allgemeine Ztirung vom 24. 1. 1983; zur Kritik s. H. Dubicl und G. Frankenberg. Entsorgung der Vergangenheir, Die Zeir vom 18.3 .1983 . 
ten die neu geschalfene demokratische Verfassung in zentralen Fragen einem ihr entgegengesetzten autoricären Bezugsrahmen. Die verfassungsrechclichen Grenzmarken wurden in Richiung der Prärogative des Staatsapparats und der Unangreifbarkeir der überkommenen Gesellschafrsordinung verschoben.

Die Entwicklungslinien dieses allgemeinen Argumentationsmusters können im Rahmen dieser Skizze nicht im einzelnen nachgezeichnet, sondern nur in Form eines exemplarischen Ausblicks angedeuret werden.

\section{Aufhebung verfassungsrechtlicher Garantien gegenüber politischen Gegnern}

Das an der rechclich möglichst ungehinderten Operationsmacht des Staatsapparats orienticrte Denken entfalcete sogleich 1950/51 in der Frage der Homogenisierung des öffentlichen Dienstes durch die Ausschaltung von Kommunisten seine Wirkung. Der Beschluß der Bundesregierung vom 20. September 1950, denzufolge die Unterstürzung der KPD und ihrer Unterorganisationen mit den Dienstpflichren eines Beamten, Angestellten und Arbeiters im Bundesdienst nicht vereinbar sei, "' wurde in richtungweisenden - die Interpretationsmaximen der sogenannten Extremistenbekämpfung der joer und soer Jahre vorwegnehmenden - Gutachten von Wilhelm Grewe und Ulrich Scheuner verfassungsrechtlich legitimiert..$^{2}$ Beide Autoren, die einst das staats- und völkerrechtliche Selbstverständnis der NS-Diktatur reproduziert und nach 194s die Identitätstheorie zur Restabilisicrung des alten Staatsapparars mit entwickelt hatren," entwarfen cinen Bezugsrahmen, durch den die Gelrung liberaler Freiheitsrechte blockiert wurde: Staatsorganisationsreche und politische Betärigungsfreiheit wurden obrigkeitlich-autoritär voneinander getrennt und das Binnenverhältnis des Staatsapparats von rechtsstaatlichen Sicherungen und grundrechtlichen Garantien weitgehend frei gehalten. ${ }^{74}$

Entgegen den Intentionen des Parlamentarischen Rates, der die Außcrkraftserzung polisischer Freiheiren der Bescimmungsgewalt der Exekutive entziehen und dem Bundesverfassungsgeriche vorbehalten wolle (Art. 18, 2 I Abs. 2 GG), "'s erkannte Grewe der Regierung eine verfassungsrechtsfreic, politische Bestimmungsmacht zur Definition und Ausschaltung des inneren Feindes* zu. Grewe argumentierce am Ende ausdrücklich sstaatspolitisch", ein anderes Wort für ein instrumentelles Verhälınis zur Verfassung, wie ein - zum Beleg notwendig längeres - Zitat zeigt:

"Dürfle ... die Zugehörigkeit zu einer staatsfeindlichen Partei zu ciner Disqualifizicrung für den öffentlichen Diensı ersı dann führen, nachdem das Bundesverfassungsgericht entschicden hat, so wäre damit dic innere Sicherheitspolitik der Bundesrepublik jedenfalls sıärkeren Hem-

71 Abgedruckt in: Deuscher Bund für Bürgctrechte (Hg.), Politische Trcuep frcht im offentlichen Diensh, Frankfurt a. M. 1951, S. :.

72 W. Grewe, Die politische Treuepliche der Angchörıgen des öffenelichen Dienstes, in: ebd., S. js ff.; U. Scheuner, Polinische Beraugung von Beamien gegen dic freiheidiche demokratische Grundordnung, ebd., S. 6 s ff.

73 W. Grewe. Der driue Warschafrskrieg. Berlin 1940, ders.. Epochen der modernen Völkerrechrsgeschıchte, Zeıtschrift lür die gesamu: Staztswissenschaft, Bd, 10; (1943). S. 260 fl. (294); U. Schcuner, Die nationalc Revolution, AäR NF 24 (1914). S. 166 ff., S. 261 lf,; ders., Die Gerichete und die Prilung politischer Suztshindlungen, RVBI. 1936, S. 437 ff.: W. Grewe, Ein Besatzungssutut für Deutschland, a. a. O. (Anm. 28); U. Scheuner, Die stastsrechiliche Xontinutiat in Deutschland, a. A. O. (Anm. I 2).

74 W. Grewe, a. 2. O. (Anm. 72), S. soff, U. Scheuner, a a. O. (Anm. 72), S. 72 ff.; ahnlich BVerfGE j9, S. 359 .

75 J. Seifern, Das Auslegungsmonopol des Bundesveriassungsgeriches bei der Verwirkung von Grundrech. Icn, in: E. Denninger $(\mathrm{Hg}$ ), Freiheitliche demokrarische Grundordnung Bd. 1, Franklur a. M. 1977, S. 225 fl. m. w. Nachw. 
mungen unterworfen, als sie in den Zeizen der Weimarer Verfassung bestanden haben. Die staatspoltuschen Nacheeile licgen auf der Hand: Die Jusciz arbeitet ihrer Natur nach norwendigeraucise langsam und wird im Wettauf mit cinem wendigen und geschmeidigen Gegner immer den Kürzeren ziehen ... Unter dem Gesichtspunkt der staatsbürgerlichen Frciheit mag es errvünscht scin, daß die Mitgliedschaft von Beamten bei einer Partei nur dann zu disziplinarischen Maßnahmen führen kann, wenn zuvor das Bundesverlassungsgerichı sein Ureeil über diese Partei gesprochen hat. Man wird sich aber überlegen müssen, ob wir uns in der Lage befinden, in der man es riskieren kann, die individuellen Rechusgarantien auf Kosten der Staatssicherbeil in solchem Maße zu versiärken. " 76

Wie die rechtsstaatliche Sperrwirkung der Entscheidungskomperenz des Bundesverfassungsgerichts für den Eingriff in die Grundrechre ausgeschaltet wurde, so wurde auch die Geltung politischer Freihejtsgarantien für Angehörige des öffentlichen Dienstes unmittelbar in Frage gestellt. Scheuners Interpretation des Diskriminierungsverbors des Art. 3 Abs. 3 GG machte dies beispiellhatr deutlich: $s$ Wenn Ar. 3 Abs. 3 GG eine Benachteiligung wegen politischer Anschauungen verbieter, so darf dieser Bestimmung keine absolure, den Wortaus isolierende Auslegung gegeben werden ${ }^{77}$ Indem das Diskriminierungsverbot, entgegen dem eindeutigen Wortlaut des Art. 3 Abs. 3 GG, nicht mehr absolut galt, konnte seine Funktion auf Null gebracht werden: „Gleichheit bedeutet nicht das Verbot unterschiedicher Behandlung. a $^{78}$ Das grundgesetzliche Diskriminicrungsverbor sollte dabei gerade den Zugriff auf politische Anschauungen als Anknüpfungspunkt für unterschiedliche Bchandlungen strikt verwchren. Scheuner hielt lediglich willkürliche Unterscheidungen und Benachteiligungen, die in dienstrechtlichen, die politische Betätigung der Beamten einschränkenden Bindungen nicht gesehen werden könnten, für unvereinbar mit dem Gleichheitssatz. ${ }^{79}$ Damit aber projizierte Scheuner die herrschende Lesart des in Art. 3 Abs. I GG normierten allgemeinen Gleichheitssatzes als eines Willkürverboes so in Art. 3 Abs. 3 GG hinein, daß dessen präzis gefaßtes politisches Benachteiligungsverbot unterlaufen werden konnte. ${ }^{80}$ Das Ergebnis von Scheuners Deduktion könnte man in letzter Konsequenz in dem Salz zusammenfassen: Das in Art. 3 Abs. 3 normierte Benachteiligungsverbor gilt hinsichtlich politischer Anschauungen nicht für Beamte.

Die von Scheuner und Grewe repräsentierte Tendenz, die Zugriffsmöglichkeiten der öffentlichen Gewalt über verfassungsrechthiche Grenzen hinaus zu schieben, die in der verwaltungsgerichtichen Rechtsprechung zum Problem der politischen Treuepflicht überwiegend mitvollzogen wurde, ${ }^{{ }_{1}}{ }^{2}$ zeigte sich auch in einem bestimmten strafrechtlichen Denken. Mit Hilfe dieses Denkens konnte die rechtsstaatlich problemarische Form der justizförmigen Ausschaltung der kommunistischen Opposition in den joer und 6oer Jahren begründet werden.

An der Position Hans Welzels, der theoretisch wohl reflektiertesten Figur der frühen

76 W. Griwe, a. A. O. (Anm, 72), S.6 1. (Hervorh, nichs im Orig.); K. Stem, Zur Verfassungstreuc der Beamten, München 1974, S. 41, Anm. 166, schloß explizit an diese Argumentscion an.

77 U. Scheuner, 2. a. O. (Anm. 72), S. 88; ahnlich wiederum BV erfGE 39, S. ${ }_{368 .}$

$7 \delta$ Ebod.

79 Ebd. Zur Knirik an der Aushahlung des polieischen Diskriminierungsverbous für Beamte vgl. entwa F. Müller, in: „Radikale“ im öffentlichen Diensı? hrsg. von H. Krirsch, B. Nagel, W. Vocgeli, Frankfurt a. M. 1973. S. 130 l.

So U.Scheuner, ebd,; zur Kritik an dieser Argumenction vgl. J. Perels, Der Gleichheitssalz zwischen Hierarchue und Demokratic, in: ders. ( $\mathrm{Hz}$.). Grundrechte als Fundament der Dernokratie, Frankfur a. M. 1979. S. 83. Daß Scheuner im NS-Siazl cinșt die Abschaffung des Gleichhuizssatzcs für Juden legutimiere, gibi seiner gegen Bcamte gerichecten Blockierung des Glrichheiessalyes des Ar. 3 Abs. 3 GG eine besonderc Nole; vg!. U. Schcuner, Der Gleichheiesgedanke in der volkisehen Verfassungsondnung, Zeitschrif́ für die gesamee Seatswissenschaft, Bd. 99 (1939), S. 267.

$8 \mathrm{r}$ A. v. Brunneck, Politische Justix gegen Kammunisten in der Bundesrepublik Deutschland 1949-1968, Erankfur a. M. т978, S. 56 m. w. Nachw. 
bundesdeutschen Stratrechtslehre, ließ sich dies exemplarisch verfolgen. Welzel hatre seinen strafrechtlichen Begriffsrahmen in ciner geistigen Parallelbewegung mit dem sich etablierenden NS-System in scharfer Frontstellung gegen die liberale Tradition der Strafrechisdogmatik enwickelt. ${ }^{8 z}$ Die Konsequenz war, daß sich die Verfügungsgewalt des Staates über die Individuen crweiterte. Die Begrenzungsfuntecion des Rechrsgüterschurzes und der ihm korrespondierenden objekriven Taubesrandsmaßigkeit suchte Welzel dadurch zu überwinden, daß er die "Akcwertc rechtlicher Gesinnung zum wesentlichen Anknüpfungspunkt für die Strafbarkcit eines Verhaltens machre. So hieß es in einem Festschriftbeirrag von 1944: "Hinter den Normen des Strafrechts stehen als ihr materialer Gehalt die Akrworte rechtlicher Gesinnung, die Treue gegenüber Volk, Reich, Führung, der Gehorsam gegenüber der Staatsgewalt. $\ll^{8}$

Welcel behielt nun nach 1945 gerade diesen Interpretationsrahmen bei, der auf eine autoritäre, grundrechtsfeindliche Struktur der öffentlichen Gewalt bezogen ist, obgleich das verfassungsrechtliche System sich fundamental gewandelt hatte. Auch uncer dem Grundgeserz, das insbesondere durch Glaubens- und Gewissensfreiheit, durch Meinungsfreiheit und das Diskriminierungsverbot für politische Anschauungen die Gesinnung zum prinzipiell sanktionsfreien Bereich erklärt, vertrat Welzel weiter seine Grundposition, das Gesinnungsmoment zum tragenden Element für die Bescimmung von Strafbarkeit zu machen. Wichriger als der Rechrsgürerschurz sei, schrieb Welzel 1950, wie beständige rechistreue Gesinnung, also die Treue gegenüber dem Staat sicherzustellen... Bloßer Rechtsgürerschutz hat eine nur negativvorbeugende, polizeilich präventive Zielsetzung, während dic zentrale Aúgabe des Strafrechts positiv sozialethischer Natur ist! ${ }^{84}$

Indem Welzel dem Gesinnungsmoment konstitucive Bedeutung für die Bestimmung der Legalität eines Verhaltens zumaß, war er in der Lage, die fragwürdige Strukcur des wesenclich gegen die KPD gerichteren politischen Strafrechts von 1951, das die rechtsstaatlich geborene Trennschärfe von Legalität und Illegalität durch die Einführung des Gesinnungsmoments verfassungsfeindlicher Absichten und Bescrebungen systematisch außer Funktion setzte, ${ }^{8 s}$ ohne Vorbehalee zu rechtfertigen. Denn die Illegalisierung objektiv verfassungskonformer Handlungen aus Gründen ihrer subjektiven Zwecksetzung fügte sich in den Begriffsrahmen Welzels nalıtlos ein. So hieß es in seinem Strafrechislehrbuch von 1954:

"An die Stelle des gewaltsamen Umsturzes - des Hochverrats im alten Sinn - ist die Zersetzung, Unıerwühlung und Aushöhlung verfassungsmäßiger Institutionen getreten, die die reale Machtergreifung auf quasilegalem Wege vorberciten... Dic Pönalisierung solcher Handlungen stößt jedoch auf große Schwierigkeiten, weil sich diese Handlungen viclfach äußerlich im demokratischen Freiheitsraum bewegen... Darum lassen sie sich weniger durch objektive als

82 H. Welzel, Nanuralismus und Wenphilosophit in Strafreche, Mannheim 193\%, S. IX. Weizel citiere den liberalen Sirufrechiler Franz v. Lisze, dem zufolge das Straireche wollwerk des Scaacsbùrgers gegenuber der seasulichen Allgewale a zu sein hat (ebd., S. 35). Dieser Gnandstruluur des Jiberalen Siralrechisdenkens hicker entgegen, daB sie $S_{12 a t}$ und Recht $\times$ niche auf die inhaltliche Fülle und Würdc ciner Idec aufbauen kannte". (cbd.) An anderer Stelle wurde deuilich, was er hierunter konkret policisch verstand: „Der Wissenschale eine polieische Aufgabee zu stellen, bedeuter ... sie . . zu den ... Natwendigkeiten der historischen Situavion zurückzuführen, die als das gegenwäruige Schicksal der vol. kisch-startlichen Gemeinschalt sie... umfüngt... n(ebd., S. 58 ). Vgl. K. Marxen. Die rechesphilosophische Begriodung der Seraftaclehre im Nationalsozialismus, in: H. Roulcuther $\left(\mathrm{H}_{\mathrm{g} .}\right)$, a. a. $\mathrm{O}$. (Anm. 56), S. 58 f. sowie allgemcin K. Marxen, Der Kampf gegen das liberale Strafrcche. Einc Studie zum Antiliberalismus der Sirafrecheswissenrchaft der 7,wanziger und dreißiger Jahre, Berlin 1975.

8) H. Welzel, Uber den substantielleo Bcgriff des Strágesetzes, in: Probleme der Strafrechuserneucrung. Fesischrift fur E. Kohlrausch, Berlin 1944, S. 109.

84 H. Welzel, Das Gesinnungsmoment im Recht, in: Festschuife fur J. v. Gierke, Berlin 1950, S. 297.

s; H. Čopic, Grundgesctz, und poliusches Seralrecht neuer Art, Tübingen 1967, S. 11; A. v. Brunneck, i. a. O. (Anm. 81), S. 74 f. 
durch subjekrive Merkmale erfassen, wie auch ganz unpolitische Delikte durch verfassungsverrätcrische Tendenz zu politischen Delikten werden. ${ }^{86}$

Ist die Begrenzung der Strafbarkeit durch das System objekriver Tatbestände, die die politische Gesinnung prinzipiell sankrionsfrei lassen, die Konsequenz der Garancie von Frcibeitsrechten, so wurde unter dem Vorzeichen der gesinnungsorientierten "posiriv-sozialechischea* Funktion des Strafrechts die stantliche Intervention in grundrechdich geschürzte Sphären politischer Freiheit systematisch ermöglicht. Daß dies bei der Anwendung des politischen Strafrechts von 195 I so weitgehend gelingen konnte, lag nicht zuletzr daran, daß richtungsbestimmende Repräsenranten der Strafrechtslehre - wie eben Welzel - ihren antiliberalen Interpretarionsrahmen ungebrochen aufrechterhielren.

\section{Identifikation von Verfassung und bürgerlicher Gesellschaftsordnung}

Die Sozialisierungsaufrräge in den Landesverfassungen von Bremen (Arc. 42), Hessen (Art. 4I), Rheinland-Pfalz (Art.61), Baden (Art. 45), Württemberg-Baden (Art. 28), ${ }^{87}$ die Sozialisierungsermächrigung des Arr. 1s GG und die weitgehende Verfügungskompetenz des Geserzgebers über die Eigentumsordnung nach Arc. i 4 Abs. I Satz 2 und Abs. 3 Satz 3 GG bildeten das verfassungsrechtliche Einfallstor, durch das die Eigentums- und Herrschaftsstruktur der bürgerlichen Gesellschaf́ potentiell gefährdet wurde. Dieses Einfallstor, das die von einem breiten Spcktrum polirischer Kräfte nach 1945 intendicrte Überwindung der gesellscharclichen Grundlagen des Nationalsozialismus ermöglichte, ${ }^{88}$ versuchte die nun einsetzende interpretative Gegenbewegung, die allerdings von einzelnen Vertrerern der alten juristischen Funkcionselite wie Hans Peter Ipsen und Theodor Maunz noch konterkarier wurde, ${ }^{89}$ weitgehend zu verriegeln. Dabei spielten zwei ineinandergreifende Argumentationslinien eine zencrale Rolle: die gegen das Vernunitrecht der Aufklärung gerichrete Form der Naturrechesrenaissance und die Umdeutung der bei Enteignungen anzuwendenden Entschädigungsklausel des Art. ${ }_{4}$ Abs. 3 Sacz 3 GG.

Die Naturrechesrenaissance zielte in ihrer konservativen Variante darauf, das Rechrsdenken der Aufklärung zu überwinden. Einschneidend unterschied sich diese Variante von der vernunftrechtlichen, wesentlich von Radbruch repräsentierten Version, ${ }^{\circ}$ die auch im Grundgeserz ihren Niederschlag fand. Gegen die Lösung des Gesctzgebers von traditionalen Bindungen und Schranken wurde auf das vorratio. nale Narurrecht zurückgegriffen, das die hierarchischen Ordnungssysteme des Feudalismus befestigte. So bezog sich Adolf Suisterhenn auf das thomistische Naturrechı," während Ernst Forsthoff aul die soziale Ordnungslehre Luthers rekurrieric $^{92}$ - jeweils zu dem Zweck, dem Gesetzgeber unüberwindbare, durch soziale Herrschafrsbezichungen bestimmte Grenzen zu ziehen. 1947 gab Forsthoff dieser Stoßrichtung offen Ausdruck. Das naturrechtliche, gegen den Positivismus gerich-

$86 \mathrm{H}$. We clyel, Das deuesche Sirairecht, a. Auf, Berlin i954, S. 356

87 Vgl. dic Darsicllung von W. Abendroth, Das Grundgeserz, 4. Aufl., Pfullingen 1973, S. 27 f.

85 Vgl, nur dic von CDU, SPD und KPD getragene Hessische Verfassung vom 1 I. Dezember 1946

89 H. P. Ipsen, Enteignung und Sozidisierung, VVDStRL to (1951), S. 74 fl.; Th. Maunz, Dic Verfassungsmäßigkeil der Hessischen Vcriassung, Wiesbaden rgsz.

90 G. Radbruch, Rechisphilasophische Besinnung (1945), a. a. O. (Anm. 18), 5. 107.

9) A Süsterhe'nn, Das Naturreche (1947), n: W. Maihofer ( $\mathrm{H}_{\mathrm{g} .}$ ), Naturreclut oder Rechtsposirivismus, Darmstade 1962, S. 16 f.

92 E. Forsthall, Zur Problemauk der Rechisemeuerung (294)), cbd., S. 79 if. 
tere Denken legitimiertc cr damit, daß es aus der Abwehr einer den Oberschichten nicht mehr stets dienstbaren legislativen Gewalt entstanden sei:

"Die Krise des Positivismus ... bcgann als der particlle Ausdruck der mit dem Zusammenbruch der Monarchie cinsetzenden Staasskrise. Dic Frage nämlich, ob das Recht wirklich allein aus der Entscheidung des staalichen Gesetzgebers hervorgehe oder ob es ein höheres Reclıt gebc, an das auch der Gesetzgeber gebunden sei, war solange eine blok theorelische, als man gewiß sein konnte, daß dieser Gesetzgeber sich nich nur mit den fundamentalen Rechtsuberzeugungen in Einklang halten, sondern auch im Detail der geserzlichen Regelung optimal gerechte Lösungen anstreben würde. Dies Vertrauen genoß der siazliche Gesetzgeber bis $1918 . \times 93$

Der gedankliche Kern der gegen eine geselischaftsverändernde Gestaltungsireiheir des demokratischen Gesetzgebers gerichteten Erneuerung des vorrationalen Naturrechts bescand darin, "nacürliche Seinszusammenhänge ${ }^{94}$ und wobjekriv gegebene menschliche Verantwortungsbereiche ${ }^{95}$ zu konstruieren, die mit den Kernelementen der bürgerlichen Gesellschaft zusammenfielen. So wurde die private Eigentumsordnung naturrechtlich festgeschrieben, ungeachtet der Sozialisierungsauferäge und Sozialisierungsernächrigungen in den Landesverfassungen, dic - in zeitlicher Parallele zur antiaufklärerischen Naturrechisrenaissance geschaffen - die Eigcntumsordnung grundsärzlich zur Disposition stellten. Übereinscimmend mir der das Gemeineigentum praktisch verwerfenden Blickrichtung der thomiscischen Naturrechtslehre ${ }^{96}$ hieß cs bei Süsrerhenn: ^Als wesentliche Garantie für die Freiheit der Person ... anerkennt der Naturrechtler die Institution des Privateigentums. "97 In entsprechender Weise bezog sich Forsthoff auf die Luthersche Ordnungsethik, welche die ständische Gliedcrung gedanklich reproduzierte. ${ }^{8}$ Er ordncte die »Insritution als überindividuelles objekrives Ordnungsgebilde des Reches", das sich im sozialen Bereich wesentlich in Eigentum, Vertrag und Erbrechr auspräge, der wbloßen Norma, die aus der Tätigkeit des demokratischen Geserzgebers cntspringt, ausdrücklich vor:

^Die Erncuerung des Rechts verlangt primär nicht neue Normen, sondern die Wiederherstellung von Ordnungen und Institutionen, ${ }^{99}$

Indem dic anciaufklärcrische Naturrechtsdoktrin die private Eigentumsordnung aus der - als positivistisch denunzienen - Bestimmungsmacht des demokratischen Geserzgebers ausgliederte, sicherte sie - bei aller gegen den Nationalsozialismus gerichteten Proklamation der Unverbrüchlichkeit persönlicher Freilheitsrechre ${ }^{100}$ die Fortexistenz der ökonomischen Grundlagen des N5-Systems. Sie erwies sich so als norwendiges, auf die kapitalistische Gesellschaftsordnung bezogenes Komplcment zur Idencitätstheorie, die das Wciterbestehen des Staatsapparats des Dritten Reiches legitimierte.

Die Grundposition der restaurativen Naturrechtsdoktrin wurde zu Beginn der soer Jahre in einer feingesponnenen verfassungsrechclichen Exegese wirksam, durch die die vom Grundgeserz festgelegte Befugnis des Geserzgebers, die private Eigentumsordnung zu verändern, durch Umdeutung blockicrt werden konnte. Dieser vielge-

93 A. A. O., S. 71 f; chenso A. Susterhenn, a. a O. (Anm. 91), S. is.

94 A. Süstcrheonn, A. a. O. (Anm. 91), S. 14.

9) E. Farsiholf, A. a. O. (Anm. 92), S. 8 3.

96 Th. v. Aquino, Ausgewahlte Schriften z.ur Staals- und Wirschaftslehre, hrsg. von F. Schregrogl, Jena $1923,5.1326$.

97 A. Susterhenn, 3. а. O. (Aлm, 91), S. 23

$98 \mathrm{M}$. Luther, Von welelicher Obrigkeic (1523), in: Luther, ausgewählt von K. C. Steck, Frankfurt a. M. $1955,5.14111$.

99 E. Forscholf, 2.2. O. (Anm. 92), S. 83, 86, 89.

100 A. Susterhenn, 4. A. O. (Anm. 9I). S. 21. 
staltige Prozeß, der von der Kaltstellung der Sozialstaatsklausel auf Verfassungsebene bis zur Identifikation von Privatrechtsordnung und Verfassungsordnung reichte, ${ }^{101}$ sei an einem strategisch zentralen Punkt verdeutlicht. Art. ${ }_{4}$ Abs. 3 S. 3 $G G$ verlangi, Arr und Ausmaß der Entschädigung bei Enteignungen unter gerechrer Abwägung der Inceressen der Allgemeinheit und der Bereiligten zu bestimmen. Diese Abwägungsklausel schließt aus, allein die Interessen des betroffenen Eigentümers zum Ausgangspunkt der Entschädigungsregelung zu machen; mit ihr war bezweckr, ein breites Spektrum von Entscliädigungsmodalitäsen zu eröffnen. ${ }^{102} \mathrm{Die}$ Einbeziehung der Interessen der Allgemeinheit schloß auch, wie Hermann v. Mangoldr, einer der der CDU angehörenden Väter des Grundgesetzes, in seinem Kommentar festhielt, die Möglichkeit ein, eine bloße Nominalentschädigung zu gewähren. ${ }^{109}$ Indem eine Entschädigung nach dem Verkehrswert nicht zwingend geboren war, konnte mit Enteignungen ein gesellschaftlicher Umverteilungseffekt erzielt werden. Die Privatwirsschaft hatte die durcly dic Norm des Arr. i4 Abs. 3 S. 3 GG entstandene porentielle Gefährơung der überkommencn bürgerlichen Eigentumsordnung klar erkannr: Die Abwägungsklausel bedeure, hieß es $1949 \mathrm{im}$ \#Indusrriekurier ", einen "Einbruch östlicher Anschauungen und Praktiken .... dessen Konsequenzen für unsere Wirtschafts- und Gesellschaftsordnung verhängnisvoll sein müssen. * $^{104}$

Durch eine u. a. von Werner Weber und Uirich Scheuner angeführe Incerpretationsrichrung wurde nun die Abwägungsklausel dadurch susgeschaltet, daß jeder Eigentumseingriff als eine Verleczung des Gleichheitssatzes angesehen wurde - mir der Konsequenz, daß dies durch die volle Entschädigung nach dern Verkehrswert ausgeglichen werden müsse. ${ }^{109}$ Mit ihrer Interprecation bezogen Scheuner und Weber unmitrelbar den Standpunkt der von Enteignungen berroffenen Eigentümer, als hätten die Interessen der Allgemeinlıeit bei der Festlegung der Entschädigungsregelung nach Art. ${ }_{4}$ Abs. 3 Sarz 3 , die ja denen der Eigentümer ausdrücklich gegenübergesteilt werden, keinerlei Erwähnung gefunden. Das weitgesteckte Ziel, die Abwägungsklausel interpretatorisch stillzulegen, war folgcndes: In Arr. is GG, der die private Eigentumsordnung hinsichelich Grund und Boden, der Narurschät»e und Produktionsmitrel zur Disposition des demokratischen Geserzgebers stellt, wird für dic Encschädigungsregelung auf Art. ${ }_{4}$ Abs. 3 Satz 2 und 3 GG verwiesen. Wenn es nun gelang, in Art. ${ }_{4}$ Abs. 3 Satz 3 GG eine Entschädigungsregelung hineinzudeuten, die auch bei der Vergesellschaftung von Produktionsmitteln stets Äquivalenzentschädigung vorschreibr, dann war die von Art. is GG für die bürgerliche Gesellschaft ausgehende Gefahr gebannt: Durch den Zwang, bei Enteignungen in jedem Fall Äquivalenzentschädigungen zu leisten, konnte der Umvertcilungseffekt von Sozialisierungen, der auch durch die Abrägungsklausel des Art. ${ }_{4}$ Abs. 3 Satz 3 GG gesicherr wurde, vereitelc werden.

101 E. Forsehoff, Begriff und Wesen des sozialen Rechessizales, VVDStRL 12 (1954). S. 8 fl.; H. C. Nipperdky, Grundreche und Privatrecht, Krefeld 1961; xur Kritik s. W. Abendroth, Die verfassungspolirische Encwicklung des Bundes, in: ders., Anugonisusche Gesellschaft und politische Demokratie, 2. Aul. Neuried 1972, S. 58 ff.

102 Vgl. hierzu eingehend: A.v. Brünneck, Die Eigentumsgarantie des Grundgesetzes, Baden-Baden 1984 , S. gof., S. 295 fi., S. 300 if.

103 H. v. Mingoldt, Das Bonner Grundgeselz, Berinn 1953, S. 103.

I04 Zit. nach W. Soergel, Konsensus und Interessen, Stuttgare 1969. S. 223.

105 W. Weber, Eigentum und Enteignung, in F. L. Neumann. H.C. Nipgerdcy, U. Scheuner $\left(H_{g}\right.$ ). Die Grundrechte, Bd. II, Berlin 1954, S. 393 : U. Scheuncr. Grundlagen und Ari der Entcignungsentschädigung, in: R. Reinhardi und U. Scheuner ( $\mathrm{Hg}$.) Verfassungsschuex des Eigcreums, Tübingen r9S4. S. 124 f. Zur Kritik s. A. v. Brünncek, 2. A. O. (Anm. 102), S. 314. 
Die restaurative Rechtslehre besaß in der frühen Bundesrepublik ein eindeuriges Übergewicht gegenüber liberalen und demokratischen, insbesondere vom Bundesverfassungsgericht vertretenen Gegenpositionen. In welchem Maße die alten Interpretationseliten in Fragen der Kontinuitär der Gesellschaftsstrukrur und des Staatsapparats dominierten, läßr sich exemplarisch daran ablesen, daß dic Anhänger der Rechusgülugkeir des Sozialisierungsarrikels der Hessischen Verfassung in der Staatsrechtslehre der soer Jahre eine ebenso verschwindende Minderheit darstellecn ${ }^{106}$ wic diejenigen, die mit dem Bundesverfassungsgericht die unter dem NS-Staat bestehenden Beamtenrechte mit dem 8. Mai r 945 für erloschen erklärzen. ${ }^{107}$ Die restaurative Rechtslehre konnte unter den Bedingungen der soer Jahre in starkem Maße schulenbildend wirken. Ihre Legicimationsgrundlagen lassen sich aber erschüttern, wenn die - dem Grundgeserz widersprechenden - antilibcralen und vordemokrarischen Wurzeln restaurativer Rechtslehre, die den Zusammenbruch des Dritten Reiches in veränderter Form überdauert haben, systemarisch bloßgelcgt werden - eine gewichrige Forschungsaufgabe.

$106 \mathrm{G}$. Wincer, Soxidisierung in Hessen 1946-1955, KJ H. 2/1974, S. 166. 107 M. Kim, a. z. O. (Anm. 3). S. 20 f.

Joachim Perels (Hrsg.)

\section{Recht, Demokratie und Kapitalismus}

Aktualität und Probleme der Theorie Franz L. Neumanns

$\leadsto \mathrm{DaB}$ derjenige, der in der Emigration die wichtigste (und in den Vereinigten Staaten auch einflußreich gewordene) Analyse des NS-Systems geschrieben hat, in der Bundesrepublik nahezu unbekannt uod in der Wissenschaft kaum rezipiert worden ist, ist symptomatisch. Franz L. Neumann ist einer der wichtigsten Denker über das Verhälınis von Recht und Politik in der ersten Hälfte unseres Jahrhunderts. Er isı die zentrale Gegenfigur zu Carl Schmilt. "(Aus der Einführung)

Die Elemente des Denkens von Franz L. Neumann, die den Zusammenhang von Recht und Kapitalismus in der Perspektive der Herstellung einer gesellschaftlichen Demokratie zum Thema haben, werden in ihren verzweigten Entwicklungslinien - einschließlich der späten Einbeziehung sozialpsychologischer Ansätze - rekonstruiert und kritisch diskutien: von dcr Weimarer Republik über die Zeit der Emigration bis in die Nachkriegsperiode. Neumanns Position wird für die gegenwärnige Diskussion über die restaurative Umformung des freiheilssichernden Rechtsbegriff́s der Aufklärungszeit fruchtbar gemacht. Die mógliche Akıualität von Neumanns Intcrpretationsrahmen tritt in den Blick.

Der Band, der eine biographische Skizze und eine Bibliographie der Schrifien von Neumann enthält, ermöglicht die - bis heuce in weitem Maße ausstehende - Auscinandersetzung mit einem der großen demokratischen Rechls- und Staatstheoreliker.

1984, 228 S., Salesia brosch., 68,- DM, ISBN 3-7890-1013-8

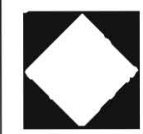

NOMOS VERLAGSGESELLSCHAFT Postiach $610 \cdot 7570$ Baden-Baden

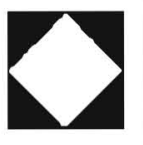

